\title{
Cancérologues et interactions de soins : quand le genre s'en mêle
}

dans «Masculinités et féminités face au cancer » (Meidani A. dir.), PUM, pp : 113-134, 2020

Introduction : le genre et le cancer

Selon l'Institut national du cancer, en 2017 , le nombre de nouveaux cas de cancers en France métropolitaine est estimé

à $\quad 400000$, dont $214000 \mathrm{chez}$ l'homme; alors que la même année, le nombre de décès par cancer est estimé à 149500, dont 84100 concernent les hommes. En parallèle, les cancers progressent jusqu'à devenir la première cause de mortalité en France, chez les hommes $\left(223.10^{-5}\right)$ comme chez les femmes $\left(114.10^{-}\right.$ 5) [Eurostat, $2014^{1}$ ]. Pandémie des sociétés contempo-raines, le cancer donne à voir la place que le genre occupe dans les interactions de soins et le façonnement genré des pratiques professionnelles des cancérologues.

L'article est issu d'une recherche des trois ans financée par l'Institut national du cancer (2014-2017) qui examine les expé-riences genrées du cancer. Ses résultats appellent à être discutés et mis en perspective à travers une revue de littérature, pour

Anastasia Meidani, Ph.D., sociologue; maîtresse de conférences en sociologie, université de Toulouse II, lisst (Laboratoire interdisciplinaire solidarités, sociétés, territoires)-cers (Centre d'étude des rationalités et des savoirs).

Arnaud Alessandrin, Ph.D., sociologue; chercheur associé au laces (Laboratoire cultures-éducation-sociétés), université de Bordeaux. Nicolas Madranges, cancérologue; Institut Bergonié.

1. http://ec.europa.eu/eurostat/statistics-explained/index.php/File :Causes_of_

death_\%E2\%80\%94_standardised_death_rate,_2014_(per_100_000_inhabitants)_YB17.png 
l'essentiel anglophone, qui interroge le couple genre/santé (Löwy, 2013; O'grady, 2005 ; Courtenay, 2000; Lorber, Moore, 2002; Duffy, 2005). Au travers de cette littérature, il apparaît claire-ment que la plupart des recherches qui portent sur la santé s'at-tardent sur les femmes et restreignent bien souvent les questions de genre aux questions de sexe. En effet, rares sont les travaux qui interrogent explicitement le cancer à partir d'une lecture intersectionnelle du genre (Meidani 2018; Meidani, Alessandrin, 2018; Braverman, 2017). À contre-pied de la majorité des études qui explorent les liens que la santé entretient avec le genre, nous soutenons que féminités et masculinités plurielles sont à saisir de manière concomitante afin de restituer toute l'épaisseur genrée des expériences cancéreuses. Plus encore, nous avançons que la prise en compte d'une orientation analytique genrée permet une compréhension plus fine des interactions de soins en cancérologie et des pratiques professionnelles afférentes.

Gardant à distance l'approche de genre, la sociologie du cancer (Derbez, Rollin, 2016) s'est longtemps intéressée aux représenta-tions relatives à la maladie, aux logiques profanes et expertes qui l'animent, aux pratiques dites à risques (et en particulier à l'alcoolo-tabagisme) et, à un moindre degré, aux risques professionnels et environnementaux qui tendent à expliquer les écarts sexués mis en évidence par des études épidémiologiques (Cohen et coll., 2015 ; Bataille, 2003 ; Legrand, Meidani, 2018 ; Herzlich, Pierret, 1984 ; Tarantini et coll., 2014). Dans ce paysage, les recherches sur les soignant.e.s en cancérologie qui développent des perspectives de genre sont quasi-inexistantes (Meidani, 2007 ; 2018). En France du moins, car dans la littérature anglophone des études ont montré que les perceptions, les attitudes et les attentes liées au genre influent sur la communication en contexte des soins (Street, 2002 ; Foss, 2002 ; Bylund, Makoul, 2002). Schmittdiel et ses collaborateurs (2000), par exemple, constatent des différences dans la satisfaction des patient.e.s qu'ils associent au genre des acteurs impliqués dans les parcours des soins. Cherchant à expliquer ces écarts de genre, d'autres travaux ont montré que les femmes, comparativement aux hommes, sont plus enclines à prendre soin de la santé familiale, ont des connaissances plus élaborées sur les questions de santé et sont accréditées d'un savoir et d'un savoir-faire reconnus comme tels, y compris par les soignant.e.s (Buller, Buller, 1987 ; Meidani, 2018).

Visant à apprécier l'impact du genre sur la dynamique inter- actionnelle de soins, notre recherche montre que la variable observée la plus centrale relève de la concordance/dissonance entre les attitudes genrées des soignant.e.s et celles des soigné.e.s. Il en résulte que les différences de genre dans les interactions soignant.e.s/soigné.e.s ont des implications importantes sur la communication en contexte des soins et, par ricochet, sur les parcours de prise en charge. En parallèle, l'analyse montre que l'approche de genre ne peut se contenter de l'étude des rapports sociaux de sexes étudiés en dehors ou au-delà d'autres facteurs sociodémographiques (âge, statut socio-économique, orientation sexuelle, appartenance ethno-raciale...), de 
la même façon qu'elle ne peut se contenter de l'étude des attitudes genrées des soigné.e.s laissant de côté celles des soignant.e.s.

Dans le droit fil de cette perspective, nous soutenons que l'ordre interactionnel de soins saisi par les concepts de la socio-logie du genre appréhendé dans un esprit intersectionnel, permet une approche plus nuancée des expériences de la maladie. Plus encore, la prise en compte du genre dans les interactions des soignant.e.s/soigné.e.s initie une lecture nouvelle des pratiques professionnelles de médecins et des parcours de soins en cancé-rologie. C'est donc au croisement de la sociologie de la santé et de la sociologie du genre que nous porterons notre regard, pour nous intéresser à la question qui compose le cœur de notre article : dans quelle mesure et de quelle manière le genre façonne-t-il les interactions soignant.e.s/soigné.e.s et par là même les pratiques professionnelles de soins de cancérologues impliqué.e.s dans ces parcours de prise en charge?

Afin de cadrer théoriquement notre approche, il convient d'ores et déjà de stipuler ce que nous entendons par "genre». Aussi rares soient-ils, les travaux sociologiques portant sur le cancer ont déjà approché la question du genre en mettant en avant les oppositions chiffrées et les stratégies différenciées mises en place par des femmes et des hommes pour faire face au cancer (Meidani, 2007; 2018). Si cette opposition apparente fait sens, les parcours des soins, divisés d'un point de vue sexué, se confrontent vite à l'éparpillement des identités et des technologies de genre (Lauretis, 2007) qui composent des féminités et des masculinités plurielles. Nous entendons ici les technologies de genre au sens de

l'ensemble des éléments qui viennent simultanément parfaire et/ ou défaire les habitudes de genre, dans un mouvement initié par l'avènement de la maladie.

En accord avec ces outils conceptuels, notre recherche ne s'engage que pour partie dans une comparaison sexuée bicatégo-rielle. En partie seulement car les subjectivités genrées se donnent

à voir elles aussi au pluriel, et devant compter sur d'autres facteurs de différenciation qui entrent en résonance avec les logiques de subjectivation de genre, comme l'âge, la génération, l'origine, le statut social et l'orientation sexuelle, supposés ou réels des acteurs impliqués dans les parcours des soins (professionnel.le.s et patient.e.s). C'est pourquoi nous entendrons ici la notion de genre comme simultanément injonctive, c'est-à-dire soumise à des principes de reproduction, mais également comme dynamique, c'est-à-dire déjà toujours susceptible d'étonner (Alessandrin, Esteve-Bellebeau, 2014).

Pour le dire en termes d'objectif de recherche, il ne s'agit pas pour nous de constituer des typologies des interactions de soins en fonction des pathologies cancéreuses considérées et étudiées par degré de gravité ni même par catégorie sexuée. Notre objectif est d'examiner ce que le genre fait au travail de soins des cancéro- logues et 
aux interactions soignant.e.s/soigné.e.s, en prenant comme point de départ le point de vue des oncologues rencon-tré.e.s $(\mathrm{N}=40)$ Tou.te.s ont été interviewé.e.s et suivi.e.s durant les trois années de terrain de l'enquête. Si le matériau observationnel recueilli (quatre cents heures d'observation in situ) oriente l'atten-tion sur les interactions soignant.e.s/soigné.e.s, d'autres facteurs entrent aussi en ligne de compte, principalement mis en mots par ces praticien.ne.s dans le cadre des entretiens. L'entreprise analytique des pratiques professionnelles plonge alors son regard dans les plis des trajectoires professionnelles, des représentations façonnées par des parcours de formation, des rencontres entre collègues le long d'une carrière, des conditions d'exercice du métier et des demandes singulières de patient.e.s, le tout éminem-ment imbriqué à d'autres caractéristiques sociodémographiques des acteurs impliqués dans les parcours des soins : oncologues et patient.e.s.

Quant au protocole d'enquête, d'un point de vue méthodo-logique, la recherche cible les cancers les plus fréquents en France 
métropolitaine, à savoir: cancer du sein, de la prostate, du poumon et du côlon/rectum. La prise en compte de l'ensemble de ces cancers permet non seulement d'élaborer un échantillon raisonné des cas par cancer, mais aussi de rendre compte des formes des rapports au genre distincts avec des cancers quasi-exclusivement féminins (sein), quasi-exclusivement masculins (prostate) et «mixtes» (poumon, colorectal).

Le dispositif qualitatif de la recherche comprend quatre cents séances d'observation in situ menées au cours des consultations, des séances de chimiothérapie, des visites dans les chambres des malades et des réunions de concertation pluridisciplinaire (rcp) dans cinq structures hospitalières situées dans trois villes du sud de la France: Toulouse, Bordeaux et Aurillac. À ces données s'ajoutent quarante entretiens semi-directifs approfondis conduits auprès des cancérologues, et équitablement répartis selon le genre $^{2}$.

Ces différents corpus des données (issues des observations et des entretiens) visent à répondre à notre problématique, ainsi qu'à tester notre hypothèse centrale sur l'interpénétration des questions de genre et des pratiques professionnelles chez les oncologues, principalement analysées dans leurs dimensions interactionnelles- en contexte de soins. Au-delà d'une simple sociographie au prisme d'une approche sexuée catégorielle, l'article revient alors sur la complexité des articulations multiples que les soins en cancé-rologie entretiennent avec le genre, revisités d'un point de vue relationnel.

Dans une première partie, nous procéderons à une analyse des interactions, des temps et des espaces où le genre s'efface. Cela signifie non pas que le genre est absent, mais plutôt qu'il agit comme une présence absente, une composante de soins invisibi-lisée, naturalisée ou bien externalisée vers d'autres soignant.e.s, pour l'essentiel les psychooncologues, des infirmier.e.s, des aides-soignant.e.s, des assistantes sociales et des esthéticiennes. Une deuxième partie sera, à l'inverse, consacrée à l'apparition du

2. En outre soixante entretiens semi-directifs ont été réalisés auprès des patient.e.s atteint.e.s par les différents cancers cités supra, et 40 entretiens auprès des proches aidants. Ce matériau n'est pas considéré dans le présent article. Ajoutons également que du côté des professionnel.le.s de santé impliquée.s dans la prise en charge de ces mêmes malades, cancérologues, chirurgiens, radiothérapeutes, chimiothérapeutes, psycho-oncologues, infirmier.e.s aides-soignantes et esthéticiennes font partie de ce corpus. Toutefois l'analyse proposée prend appui sur la population des oncologues.

genre dans les pratiques des cancérologues et dans leurs inter-actions avec les patient.e.s. Nous montrerons alors que l'émer-gence du genre par les soignant.e.s ne se déploie pas de la même façon dans les trajectoires de la maladie qui se conjuguent au féminin et au masculin, et que les patient.e.s dont les attitudes transgressent les normes stéréotypées de genre, perturbent les attentes des oncologues. Notre troisième partie reviendra sur la dynamique du genre dans les interactions de soins faisant place aux patient.e.s. Puis, l'article distinguera ces oncologues pour qui le souci du genre semble être apparu en amont de notre rencontre ou qui, du fait de leur implication dans la 
recherche, ont fait leur ce concept. Quelles sont les caractéristiques de ces soignant.e.s?

Là où le genre s'efface

En contexte de santé, le genre avance masqué. Partout présent, des formations jusqu'aux suivis médicaux en passant par la distri-bution des carrières de soignant.e.s et la communication médicale en contexte de soins, le genre n'est cependant que très rarement nommé durant les parcours professionnels de ces praticien.ne.s. Si les sciences humaines et sociales ont été nombreuses à se pencher sur le couple genre et médecine (ou genre et santé), du point de vue des soignant.e.s, cette grille de lecture reste bien peu mobilisée dans leurs parcours de formation. Sur le plan pratique, lorsque les médecins traitent des patient.e.s, ils/elles ne se penchent que très rarement sur la gamme complète des pratiques et des repré-sentations de genre. Cherchant plutôt à voir si la case «homme » ou «femme » est renseignée, les oncologues ne s'attardent sur cette question que lorsque l'orientation sexuelle du malade vient perturber les rituels communicationnels des pratiques des soins. Nous y reviendrons. Par conséquent, le genre est souvent opérationnalisé comme «sexe» dans les milieux de soins en cancérologie.

L'effacement du genre dans leurs pratiques se justifie alors par d'autres éléments, plus ou moins explicitement exprimés. À l'évocation de notre objet de recherche, les cancérologues rencontré.e.s mettent rapidement en avant quatre motifs d'éloi-gnement au genre : la temporalité de soins bien souvent dictée par l'urgence et le caractère protocolaire de la prise en charge, le

renvoi du genre du côté du care, la neutralité supposée acquise du rôle des soignant.e.s face à la question du genre de la patientèle et, enfin, le manque de formation. Ces différents éléments ont pu être mobilisés séparément ou conjointement, mais tous apparaissent assez nettement dans les discours des oncologues, hommes ou femmes, professant dans les établissements de soins considérés dans le cadre de la présente enquête. Revenons sur chacune de ces justifications en commençant par la dernière.

\section{Genre et formation en cancérologie}

L'ensemble des soignant.e.s interviewé.e.s s'est interrogé,

à divers degrés, sur l'approche théorique de notre recherche en tentant de définir le terme de genre: «Par genre, vous entendez quoi?» Si le passage réductionniste du propos au couple femme/ homme fut maintes fois exploré, le fait que le genre n'en soit pas toujours strictement le synonyme a été vite acquis. "J'ai lu un de vos textes», nous confie Pierre ${ }^{3}$ un jeune cancérologue de 38 ans. «En fait si j'ai bien compris ce que vous dites, c'est qu'il y a le sexe 
anatomique et puis il y a de la masculinité et de la féminité qui se répartissent différemment en chacun de nous, c'est ça ${ }^{4}$ ?», poursuit-il. Si la question du genre n'est pas évidente aux yeux des professionnel.le.s enquêté.e.s, elle est toutefois acceptée comme matériau de discussion entre nous: équipe de chercheur.se.s et soignant.e.s.

À la suite des travaux de Joëlle Kivits et ses collaborateurs (2013), l'analyse proposée confirme que le temps d'acclimatation mutuelle passe par le partage de concepts et de sens entre méde-cins et sociologues. Le constat d'un apprentissage réciproque laisse alors entrevoir une première limite dans l'appréhension de la notion de genre : celle de la formation initiale de ces professionnel. le.s. «Nous n'en avons jamais entendu parler. Enfin, on a étudié les différences entre les femmes et les hommes, mais nous n'uti-lisons pas le concept de genre», nous précise Stéphanie, 42 ans. "C'est bien qu'on en discute avant parce que dans nos études

3. Conformément aux règles d'anonymisation, tous les prénoms mobilisés correspondent à des pseudonymes.

4. Les données brutes communiquées au long de l'article renvoient indistinctement à des extraits d'entretiens formels ou informels, réalisés dans le cadre des interviews ou des séances d'observation.

nous n'y avons pas été confrontés », complète Benjamin, 47 ans. Si nous n'avons pas eu accès au volume horaire des différents cours et options disponibles à l'époque des parcours de forma-tion des soignant.e.s enquêté.e.s, nous nous sommes procuré.e.s celui de l'année 2016. Ainsi l'université dans laquelle Stéphanie a fait ses études ne fournit toujours aucun cours d'introduction aux sciences humaines et sociales abordant les études de genre, et limite le sujet des inégalités aux questions de classes et de territoires. Quant à l'université qui accueillit Pierre, vingt ans aupa-ravant, elle offre deux heures sur le genre, en première année de médecine. Comme le soulignent les travaux de Céline Avenel (2012) sur les formations en médecine, la question du genre n'est pas encore entrée dans ces cursus.

\section{La temporalité de soins : entre urgences et protocolisations}

Au-delà des formations initiales, c'est le type même de soins effectués qui justifie, selon les oncologues, l'absence de considéra-tion de toutes les questions en lien avec le genre. «Vous comprenez, on a affaire à des patients qui ont une pathologie grave. Il y a l'ur-gence des patients mais il faut également gérer celle des familles » (Georges, 50 ans). De fait, le temps moyen d'une consultation est très variable d'un établissement de soins à un autre. Dans les centres hospitaliers urbains étudiés, le temps consacré aux patient.e.s n'excède que très rarement le quart d'heure (hors consultation d'annonce). Au contraire, dans les instituts, tout comme dans les établissements situés en zone rurale, il s'écoule facilement 
plus de vingt minutes par patient.e. Ces différences s'expliquent principa-lement par les moyens humains et les ressources financières mis à disposition du personnel. Il en résulte que moins les professionnel. le.s ont le temps d'échanger au-delà des aspects cliniques avec les patient.e.s, plus la question du genre, et avec elle celles du corps et des émotions, passent sous silence.

«Ce sont des questions que j'aborde peu avec mes patientes. Peut-être que je devrais les aborder mais, vous le voyez bien, on manque de temps, donc on s'attarde sur l'essentiel : les résultats des examens », résume Philippe, 45 ans.

Son collègue Pascal, 53 ans, complète à son tour le propos : «Quand une patiente vient me voir, que je dois lui annoncer qu'on vient de

lui diagnostiquer un cancer [du sein], ou même après, lorsqu'on doit la suivre durant une chimiothérapie, on a d'abord le droit à des questions d'ordre vital. Ce qui vous intéresse [le genre], ça vient après, et pas forcément avec nous d'ailleurs. »

Les recherches en sciences humaines soulignent pourtant que face à la maladie, les émotions et les craintes (notamment celles liées aux actes chirurgicaux, aux effets iatrogènes du traitement ou aux relations de confiance avec les soignant.e.s) sont des véhicules du genre (Meidani, 2008; 2018). Dans la continuité de nos travaux précédents, nos observations montrent que le genre est partout, y compris lors des consultations d'annonce. Dès lors, où se loge ce genre qui n'est pas pris en compte ? Une voie d'externalisation privilégiée émerge dans les parcours de soins, le genre est alors transféré vers d'autres spécialités (para)médicales, présentes (ou pas) aux établissements de soins. Cela a pour corollaire de consi-dérer que la fonction du soin en cancérologie subsume le genre, et que les demandes qui émanent de ce côté-ci du soin sont de l'ordre du «particulier», et bien souvent du féminin, ou alors de l'ordre du «spécifique», entendu ici comme le signe propre d'une identité homosexuelle, bisexuelle ou, plus rarement, transgenre.

\section{Quand le soin entre en concurrence avec le genre: le mythe du traitement égalitaire}

Le dogme des traitements théoriquement neutres réservés aux patients, ne résiste jamais longtemps à l'épreuve du terrain. Et pourtant les médecins rencontré.e.s y croient communément. Le champ lexical de l'indifférence revient alors souvent au galop: «Je ne fais pas de différence entre les hommes et les femmes » (Sabrina, 47 ans) ; «Je ne vois pas de différence» (Georges, 50 ans) ; «Le suivi est protocolisé. Tous les patients ont le même ou presque» (Louisa, 52 ans) ; "Un cancer est un cancer, peu importe le sexe» (Philippe, 45 ans). Pourtant, ce mythe d'une égalité de traitement des corps, indépendamment de leur identité de genre, a déjà été battu en brèche dans l'analyse d'autres sphères institutionnelles, comme celle de l'école. En paraphrasant la thèse de François Dubet (2017) concernant l'égalité des chances, nous soutenons que l'indifférence au genre est un «mythe nécessaire» pour de 
nombreux.ses soignant.e.s qui visent à (voire espèrent) traiter, à l'identique, tou.te.s leurs patient.e.s.

Or, du point de vue des patient.e.s et de l'adhésion théra-peutique, la prise en compte des éléments relatifs au genre de la personne n'est pas anodine. Et pour cause... Hommes et femmes n'ont pas le même taux de compliance aux soins. Tout se déroule alors comme si la fonction soignante était investie par les profes-sionnel.le.s d'une force a-genrée. Présentée comme la condition d'un «bon» traitement, la neutralité est supposée acquise.

«Je ne connais pas de médecin, sauf peut-être des vieux oncologues,- qui traitent différemment les femmes et les hommes » (Pierre, 38 ans).

«Dans ma pratique, je ne vois quasi exclusivement que des femmes. Mes patients atteints de cancer du sein sont assez rares. Je ne crois pas qu'il y ait une spécificité de ma pratique en ce qui concerne les hommes » (Laurent, 47 ans).

Dans un tel contexte, la neutralisation du genre à l'œuvre procède par un double effet d'externalisation des toutes questions genrées aux métiers du care (dont s'excluent les cancérologues); et de localisation du genre aux organes sexués. Si l'intervention iatrique agit dans ce cadre «contre » la prise en compte de l'iden-tité du genre de la personne malade, nous verrons qu'il s'agit surtout d'une éviction fictive. En effet, dans la mesure où il n'y a pas d'extérieur au genre, le soin lui-même est pleinement compris dans le processus des normes et des polices de genre.

\section{Care : externalisation des soins, externalisation du genre}

Àl'exception des actes techniques (ablations, fonctions sexuelles et reproductives, chirurgies réparatrices), lorsque le genre se fait pressant, au nom de la féminité ou de la masculi-nité du/de la patient.e, une procédure d'externalisation s'opère. «J'envoie mes patientes voir la psychologue si j'estime que c'est nécessaire. Je ne suis pas là pour traiter les questions d'ordre psychologique. Peut-être que d'autres le font mais moi je ne le sens pas » (Léa, 49 ans). Les spécialistes de la question ont déjà montré que le care et le cure ne sont, symboliquement et écono-miquement, pas distribués de la même façon dans le domaine du soin (Molinier et coll., 2009 ; Brugère, 2017). L'imbrication du care avec les questions de genre est également travaillée en ce sens que tout ce qui renverrait à la sollicitude serait non seulement péjoré 
et minimisé, mais également poussé vers le féminin. Certain.e.s soignant.e.s expriment même une certaine exaspération des demandes des patient.e.s qui se situent dans ce registre: "C'est un peu ce que j'appelle de la bobologie, les aphtes, la chute des cheveux et compagnie » (Roland, 49 ans); "J'évite de dire que ça fait mal, l'objectif c'est le traitement [...] Si l'on exhaussait toutes les demandes particulières de tous les patients on y arriverait pas » (Pascal, 57 ans). L'extrait ethnographique suivant confirme ce même constat qui se retrouve aussi dans certaines demandes de soins que Claude, 53 ans, commente ainsi :

« Il y a des patients qui viennent vous voir avec des demandes parti-culières. On a des patientes qui nous demandent des conseils sur des médecines alternatives, comme la dame que vous avez vue ce matin. Qu'est-ce que je peux leur dire, si ça leur fait du bien... Mais spontanément, non, je ne le propose pas. Ici, on dispose des tech-niques et des méthodes, d'un savoir-faire, qui se passe de ça [soins alternatifs].»

Dans les cas présents, le genre renvoie vers certaines représen-tations du féminin qui doivent être «traitées» par un tiers, profes-sionnel.le ou aidant.e informel.le. La cancérologie, quant à elle, demeure toujours à l'égard du genre dans un «certain universel ».

Le genre ou la préférence pour le sexe?

Lorsque les questions de genre ne sont pas sous-estimées, elles se cristallisent bien souvent en un endroit précis du corps. Le souci du genre réside alors en la localisation de l'organe affecté par la tumeur ou les effets iatrogènes du traitement. Ici, le cancer du sein et le cancer de la prostate offrent des illustrations édifiantes. "Pour les patientes que je suis, la poitrine et les cheveux sont des enjeux importants » (Philippe, 45 ans). Une différence s'opère alors entre les questions médicales et les autres préoccupations, externali-sées à d'autres soignant.e.s : «Pour les perruques et les postiches je renvoie à l'esthéticienne du centre», poursuit Philippe. Il ne s'agira pas de dire que cette interrogation sur le «lieu» du genre est illégitime: réductrice, certainement. Soulignons toutefois que, parfois, les patient.e.s eux/elles-mêmes délimitent ce qui entre en jeu dans leurs masculinité et/ou féminité durant les parcours de soins, afin de circonscrire leurs demandes et les adapter aux

profils des soignant.e.s impliqué.e.s dans leur prise en charge. D'autres fois, nous le verrons, cette limitation du genre à certains organes sexués s'émiette, mettant à l'épreuve les représentations des soignant.e.s mais aussi, dans une certaine mesure, celles des soigné.e.s et de leurs proches. 
Femmes et hommes se distinguent assez nettement dans l'ap-proche qu'en ont les cancérologues lorsqu'il s'agit de "palier », « réparer», "masquer» (les termes varient selon les patient.e.s et les praticien.ne.s), les traces de la maladie. Pour les patientes dans les cas des cancers observés, la dimension esthétique est très vite mise en avant de part et d'autres. Camille, 45 ans, rend ainsi compte de ce constat: "J'avertis toujours mes patientes de la chute des cheveux et je leur demande comment elles vivent cela, si quelqu'un est là pour les épauler : c'est important pour les femmes les cheveux, c'est un signe social fort. » La littérature sur l'expérience de la maladie cancéreuse est d'ailleurs abondante à cet endroit du genre, et souligne les stratégies d'adaptation multiples que les femmes mobilisent afin de faire face aux conséquences de la chimiothérapie.

Quant aux effets cutanés des traitements, pareillement visibles chez les femmes et les hommes (à type et durée de traitement égaux), le champ lexical des soignant.e.s diffère. Ainsi face aux patientes, le vocabulaire de l'esthétisme apparaît plus souvent que celui de la dermatologie ou encore de la perte de poids, essentielle-ment réservé à la patientèle masculine. Plus encore, face au couple soigné.e/aidant.e, les termes du/de la soignant.e varient, et l'extrait ethnographique qui suit en atteste :

«Quand une patiente vient me voir après une chimiothérapie, j'exa-mine les effets du traitement sur la peau. Je conseille des crèmes spéciales et quand monsieur accompagne madame, comme ce fut le cas tout à l'heure [ce qui est plus rare que l'inverse], je rappelle que des esthéticiennes sont aussi là pour les aider à se sentir mieux. C'est important dans le couple car le cancer chamboule beaucoup de choses, notamment la libido » (Pierre, 38 ans).

Si la localisation du genre à tel ou tel endroit des corps fémi-nins finit par les objectifier, il en va de même, sur un registre

sexuel, pour les corps des hommes. Mathieu, 41 ans, suit des patients atteints de cancer de la prostate. Il travaille de concours avec un urologue. Lors de la pause déjeuner qui suit la matinée d'observations, tous deux commentent ainsi les parcours de patients observés :

«Pour les hommes bander c'est hyper important. La continence aussi c'est important. Mais le sexuel c'est central, car dès qu'il y a impuissance physique, il y a perte de l'estime de soi et négligence dans le traitement.

- Moi j'ai des hommes qui viennent me voir en me disant : “Écoutez docteur, j'ai passé l'âge des rapports sexuels mais c'est ma femme qui veut." Alors je leur propose des injections [intracaverneuses], pour qu'ils puissent satisfaire les désirs de leur compagne. »

Cette tentative de localisation du genre ne jaillit pas de nulle part. Elle procède des deux phénomènes conjoints. D'un côté, le souci des soignant.e.s de circonscrire la demande afin de mieux la traiter, au risque d'en passer d'autres sous silence. De l'autre, les demandes des patient.e.s relatives à une continuité de genre («être comme avant $» /\langle$ pouvoir faire pareil qu'avant »/ « recommencer à... »), signe par excellence d'une rémission qui se veut totale. 


\section{Le genre comme allié silencieux}

Du côté des soigné.e.s comme du côté des soignant.e.s, il s'agit bien souvent d'assurer une continuité entre l'avant et l'après-- traitement, afin de limiter l'effraction que constitue la maladie. Ce souci de «mêmeté » trouve dans le genre un allié de taille, puisque c'est aussi dans cette quotidienneté, ces habitudes corporelles, sexuelles ou relationnelles (le partage des tâches domestiques dans le couple, par exemple), que se joue la capacité à (faire) accepter la maladie, le traitement et ses conséquences. De nombreux cancéro- logues s'appuient sur les éléments de genre communiqués par les patient.e.s, dans le but de les rassurer tantôt de leur capacité à résister, tantôt de l'importance toute relative de l'irruption cancer.

«Vous verrez, vous pourrez faire comme avant» (Camille, 38 ans).

«Vous faites toujours votre jardin?» (Philippe, 45 ans).

«Votre femme est-elle toujours une aussi bonne cuisinière ?» (Nadine, 44 ans).

«Sexuellement, ça se passe mieux depuis le traitement que je vous ai donné ? Vous retrouvez un peu votre libido ? (Claude, 53 ans). «Au niveau de la fatigue, ça disparaît un peu j'ai l'impression, non... » (Bastien, 41 ans).

$\mathrm{Si}$, comme nous avons pu le souligner préalablement, le genre avance masqué, toutefois, pléthore des praticien.ne.s « font du genre sans le savoir», en ce sens que leur regard ne se limite jamais totalement aux organes. Au-delà de la réflexivité des médecins au sujet du genre, la question qui demeure est justement ce qui différencie les soignant.e.s qui intègrent, plus ou moins sciemment, le genre dans leurs consultations, et celles et ceux qui y résistent sans jamais s'y soustraire complétement.

\section{Quand la localisation du genre s'émiette}

Avant de rendre compte de l'émiettement du genre dans les interactions des soins en cancérologie, précisons toutefois que depuis le début de cette contribution nous nous situons exclusi-vement en régime hétérosexuel et cisgenre. C'est-à-dire qu'il n'a jamais été question jusque-là des soignant.e.s et/ou patient.e.s homo- bisexuel.le.s ou trans que nous avons pu rencontrer dans nos différentes recherches (Meidani, Alessandrin, 2017). Or, la question du genre s'impose directement dans les interactions de soins lorsque les normes de genre sont brouillées plus frontale-ment encore par des parcours de genre que par la seule maladie.

Deux mouvements dissonants se font alors jour dans les attitudes des praticien.ne.s. Le premier, à l'image de ce que nous venons de voir, neutralise les caractéristiques individuelles: «Je traite tous mes patients de la même façon, homo ou hétéro, ça ne 
fait aucune différence pour moi», nous rassure Régis, 57 ans. Mais d'autres cancérologues préfèrent, là encore, externaliser cette question, d'autant plus lorsque la sexualité ou l'identité de genre du/de la malade ne sont pas attendues : «C'est vrai que pour les questions plus personnelles, moins médicales, j'envoie directe-ment vers le service de psychologie » (Hervé, 52 ans). À cet égard, l'effet génération, plus encore que l'effet âge, semble jouer un rôle important dans les représentations des soignant.e.s et les postures plus ou moins transgressives des «plus jeunes» de ces médecins, parfois directement concerné.e.s par l'homosexualité.

\section{L'intimisation du genre}

Lorsque les établissements de soins font place au genre, c'est souvent sous le signe d'un processus de privatisation des formes de ses expressions. Le genre qui nous a été livré relève alors non pas des rapports sociaux mais de la sphère privée et des relations interpersonnelles. Si dans les soins en cancérologie, le genre est classé dans la case de l'intime, c'est parce que l'offre institution-nelle peine à lui faire place. Les modalités organisationnelles des dispositifs de prise en charge en cancérologie, la coprésence d'une multitude de professionnel.le.s dans les parcours de soins, le prin-cipe d'autonomisation des patient.e.s, etc., donnent un aperçu des limites de l'offre institutionnelle. Le processus de privatisation qui en découle passe alors par le transfert de cette composante du travail de soins vers des espaces ordinaires de santé.

Ainsi confiné à l'espace privé, le genre ne peut espérer obtenir ni reconnaissance ni réparation, aussi malmené soit-il par l'avè-nement de la maladie et le protocole de sa prise en charge. Tout se passe alors comme si le système de soins, par cette privatisation du genre, n'était pas en mesure de considérer les dommages causés à ce dernier. Présents certes pour soi, ces dommages ne peuvent exister pour autrui, alors qu'ils restent dans l'ombre pour les médecins et le système de soins. Ce faisant, l'institution tient

à

distance toute forme de politisation de l'intime qui convoque-rait le genre, considéré ici comme une entité fixe et stabilisée. Et pourtant, le genre face au cancer n'incarne rien d'immuable. Il se transforme à l'aune de la trajectoire de la maladie, travaillé non seulement par les caractéristiques cliniques de la tumeur mais aussi par les normes culturelles qui le guettent et auxquelles il est sommé de répondre.

Prenant acte de l'enjeu, les professionnel.le.s placent le genre dans l'intime.

"C'est difficile de rentrer dans cette sphère de l'intime, de la sexualité ou de ce que vous appelez le genre. Si le patient aborde la question je le suis, sinon je préfère ne pas brusquer les choses » (Stéphanie, 42 ans). 
«Je ne souhaite pas offusquer car ces choses relèvent du privé. Les questions de reconstruction mammaire ne sont pas faciles à aborder. Les patientes viennent et me disent : "Guérissez-moi,

docteur, guérissez-moi”, sous entendant que le reste on s'en fout. Parfois je leur dis : “Oui, aujourd'hui ce n'est pas votre priorité mais demain ?" »(George, 53 ans).

Ces extraits nous montrent que nous n'avons pas affaire à une simple opposition entre les pratiques et les représentations de la sphère privée et celles prescrites par la sphère publique de soins. Loin d'une telle opposition, ce qui se joue dans les parcours de soins relève plutôt d'un maillage qui se recompose et qui conduit chacun.e des acteurs et des actrices impliqué.e.s à l'élaboration de sa propre morale du genre.

\section{Le souci du genre}

Comme nous avons pu l'observer, la prise en compte du genre dans les interactions soignant.e.s/soigné.e.s ne relève pas de l'évi-dence. Scrutées au prisme du genre, les interactions de soins se montrent plurielles - du moins sous le regard des praticien.ne.s. Mais cette pluralité se retranscrit également dans les apprécia-tions des patient.e.s. Dans cette dernière partie, nous souhaitons revenir sur la manière dont le genre des patient.e.s interfère sur les interactions de soins, mais aussi sur les caractéristiques des soignant.e.s qui nous ont accueilli.e.s, en interrogeant ce qui fait d'elles ou d'eux des praticien.ne.s plus ou moins soucieux.ses des questions de genre : est-ce dû à leur âge, à leur formation initiale, au service dans lequel ils/elles professent ou bien encore à leur propre parcours de genre? Pour poursuivre, nous reviendrons sur une caractéristique commune à l'ensemble des cancérologues qui expriment un souci du genre : l'ergonomie de leurs consultations.

\section{Interactions de soins et genre : lorsque les patient.e.s s'en mêlent}

L'étude des interactions soignant.e.s/soigné.e.s convoque le genre des malades jusquelà resté en retrait dans l'analyse, au profit d'une réflexion centrée sur les cancérologues et les liens qu'ils/elles entretiennent avec cette question. Si cette entrée large-ment sous-documentée dans les recherches disponibles assoit la légitimité thématique de cette contribution, à présent, nous nous proposons de rendre compte de la place qu'occupe le genre des patient.e.s dans la dynamique interactionnelle. Les données 
observationnelles montrent la persistance des rôles sexo-spéci-fiques dans les établissements de prise en charge, et soulignent des différences genrées significatives qui guident le rôle du/de la malade et animent les interactions de soins (Foss, 2002). Trois éléments peuvent rendre compte de ces écarts sexués observés lors des consultations.

Tout d'abord, et du côté de la patientèle, les responsabilités traditionnelles qui incombent aux femmes en matière des soins familiaux peuvent les amener à avoir des attentes plus «élevées » face aux professionnel.le.s de soins, creusant de la sorte leur insatisfaction quant aux services procurés. Par conséquent, elles peuvent se montrer plus critiques envers les médecins, notamment lorsque leur attitude déroge aux attentes sexo-spécifiques tradi-tionnelles. L'absence d'empathie de la part de certaines médecins cancérologues est à ce titre significative, et peut activer l'anathème de ces patientes. Par ailleurs, lors des consultations, ces dernières restent en attente d'une communication qui prend en compte leur mode de vie, leur profil social et des questions d'émotivité orien-tant de la sorte leurs expectatives, ce qui les rend souvent plus exigeantes que leurs homologues hommes. Raphael, 39 ans, nous confie: "Certaines d'entre elles ont vite fait de tomber dans la caricature : "Oui c'est un jeune, macho, sexiste." C'est plus simple avec les hommes [malades]. »

D'autre part, ayant généralement plus de connaissances sur les questions de santé que la patientèle masculine, les femmes sont souvent amenées à discuter plus en détail leurs problèmes de santé. Elles ont, par exemple, tendance à présenter plus d'in-formations personnelles sur leurs antécédents et à décrire plus longuement leurs symptômes, ce qui les place dans l'attente de plus d'informations de la part des médecins. En outre, la patien-tèle féminine peut se montrer plus consciente que les hommes de l'impact de certains facteurs sur leur capital santé (comme les conduites dites à risques), la faible activité physique et sportive, le manque d'attention prêtée aux besoins psychosociaux ou encore l'absence de pratiques préventives (Foss, 2002; Ahmed, Bates, 2007). Cet extrait ethnographique avec la chef de clinique en cancérologie digestive, une médecin de 47 ans, confirme l'analyse observationnelle :

«Certaines d'entre elles, généralement d'un niveau de vie élevé, ont une bonne hygiène et sont conscientes des dégâts de la consommation tabagique, font attention à leur alimentation, exercent une activité physique régulière, et sont ponctuelles en matière d'examens. Mais elles sont aussi en attente d'une attention particulière, de plus d'informations, etc. »

Par ailleurs, lors des consultations observées, la patientèle féminine a tendance à exprimer davantage ses ressentis, est plus attentive aux indices non verbaux de la 
communication avec les médecins, et montre une préférence pour des relations qui la soutiennent émotionnellement et qui s'apparentent à des configurations interactionnelles hiérarchiquement «plates». En ce sens, ces femmes sont susceptibles de créer des opportunités empathiques plus intenses d'un point de vue émotionnel (Bylund, Makoul, 2002). Lors de la séance de débriefing qui clôt une journée d'observation dans le cabinet de Nicolas, spécialiste du cancer du sein, ce dernier annote comme suit la dynamique interactionnelle de soins :

«Généralement jouer au petit chef ça ne marche pas. Il vaut mieux chercher à les convaincre. Les femmes sont plus sensibles au langage du corps [...] lorsqu'on leur pose la question: "Comment vous vous sentez?", tout remonte et nous assistons souvent à des scènes intenses d'un point de vue émotionnel. »

Pourtant, et alors que les patientes participent plus activement que la patientèle masculine aux consultations médicales, leurs préoccupations sont souvent perçues par les médecins comme moins importantes que celles des hommes. Ainsi, bien qu'elles demandent davantage de temps et d'explications de la part des cancérologues qui les suivent, ces professionnel.le.s ont tendance

à leur adresser des réponses plus courtes et moins techniques, comparativement à celles réservées à la patientèle masculine. Raphael, spécialiste des cancers du poumon, rend ainsi compte de nos observations que nous lui soumettons : «Peut-être que je prête plus d'attention aux hommes côté observance, car ils sont moins assidus que les femmes. Donc j'insiste davantage et donne peutêtre plus d'informations techniques si le malade en demande.» Et pourtant, les patientes interviewées valorisent nettement plus les compétences techniques des soignant.e.s que les hommes malades (Schmittdiel et coll., 2000), même si elles

n'inscrivent pas les échanges dans ce registre lors des consulta-tions-comme le font habituellement les hommes. Inversement, alors que la patientèle masculine peine à décrire ses symptômes et plus généralement son expérience de la maladie, non seulement les hommes obtiennent plus d'attention de la part des médecins (Meeuwesen et coll., 2002) mais, de surcroît, leurs préoccupations sont davantage prises en compte que celles de la patientèle féminine (Ahmed, Bates, 2007).

Pour conclure avec cette partie, les patientes, bien plus que les patients, accordent une grande importance aux différents aspects relationnels de soins et se montrent plus affectées par une impli-cation moindre dans les processus décisionnels en lien avec leur protocole de prise en charge (Foss, 2002). Dans la suite logique de ces constats, les patientes auraient davantage propension

à

porter des jugements négatifs sur les soignant.e.s dont l'atti-tude jugée autoritaire pourrait laisser entendre que les décisions médicales qui les concernent ne sont pas partagées. En consé-quence, les femmes se montrent généralement moins satisfaites de la rencontre clinique que les hommes malades, et 
sont plus susceptibles de changer de médecin en raison de leur insatisfac-tion. Benjamin, 44 ans, décrit ainsi les nouvelles arrivées dans son cabinet lors des consultations matinales :

«Oui, il y a des patientes, comme ces dames ce matin, qui viennent me voir parce que leur praticien ne leur convient pas et elles ont entendu parler de moi, essentiellement par des proches suivies dans l'institut. Et il y en a aussi d'autres qui ont voulu changer de praticien à cause de mon homosexualité. »

\section{L'effet génération, de genre et de sexualité chez les soignant.e.s}

À l'opposé de ce qui a été présenté jusque-là, les résultats qui suivent ont le statut non pas de preuve mais d'indice. En accord avec cette approche indicielle, nous nous proposons de déployer certaines pistes pour interpréter le fait que certain.e.s cancérologues ont, ou n'ont pas, répondu à nos sollicitations puis, lors des observations, ont - à divers degrés - intégré la question du genre. Premièrement, il semble que l'effet «formation initiale» soit peu efficient pour expliquer l'implication de médecins à nos côtés. Comme nous l'avons vu, les formations premières n'injectent

pas, ou peu, la question du genre dans les cursus. En revanche, la formation continue est souvent évoquée: «Je travaille avec un sexologue, alors vos questions m'intéressent» (Pierre, 38 ans), «Je fais un du [diplôme universitaire] sur l'hypnose. Les questions sociales doivent nous interpeller, nous les médecins » (Benjamin, 47 ans). Plus encore, ce sont des rencontres qui confrontent les praticien.ne.s à des expériences restées sans réponse et qu'ils/elles espèrent aborder à nos côtés :

«J'ai eu une patiente transgenre un jour. J'ai vu que vous aviez travaillé dessus, ça m'intéresserait d'en discuter avec vous » (Benoît, 40 ans).

«J'ai des couples d'hommes qui viennent me voir. Moi je suis hétéro, donc je ne sais pas si je dis toujours bien les choses. Vous avez sûrement un regard neuf à m'apporter » (Benjamin, 47 ans).

Mais, au total, plus que l'effet genre des praticien.ne.s (et la question de sexualité qui va de pair), c'est la génération et l'âge de ces dernier.e.s qui semblent faire la différence. En effet, sur l'ensemble des cancérologues suivi.e.s, la majorité écrasante avait moins de 45 ans au début de la recherche. De l'avis même des soignant.e.s, la génération plus que l'âge modifie beaucoup la sensibilité aux questions de genre: «Les vieux cancérologues, tu les verras jamais prendre du temps avec toi pour discuter sexualité ou féminité » (Pierre, 38 ans); «Dans ma carrière, je ne me souviens pas que l'on m'ait formée à cela. Ni à la fac ni durant mes années d'internat. Tu imagines les anciens...» (Stéphanie, 42 ans). 
Au long de nos observations, nous avons pu constater que, en régime de soins, lorsque le souci du genre advient, il épouse alors la préoccupation de la bientraitance. Ce glissement s'opère dans la gestion de l'espace et du temps de soins, du moins en ce qui concerne les cancérologues les plus sensibles aux interpellations de genre des patient.e.s. Le souci du genre se rend alors visible dans la position des corps des soignant.e.s et l'ergonomie de la consultation. C'est la prise en charge du cancer du sein par des hommes cancérologues qui fournit les illustrations les plus exemplaires à ce propos.

«Je ne vois pas comment on peut expliquer à la patiente les diffé-rentes options dans le traitement avec les différents effets secondaires et les succès relatifs à chaque technique, si on est loin [physiquement] d'elle [...] Oui c'est vrai que je prends toujours le petit tabouret, pas la grande chaise en cuir. Comme ça je peux me déplacer à côté d'elles et leur faire un schéma pour résumer le parcours que je leur propose ", avance Benjamin, 47 ans, incité par l'enquêtrice à rendre compte de ses pratiques, maintes fois observées dans son cabinet.

Les propos de Stéphane, 56 ans, abondent dans ce même sens quand nous l'interrogeons sur «ses manières de faire », l'invitant à déployer la logique qui soustend ses pratiques de routine:

«Nous, on a un savoir. Mais ça ne sert pas à grand-chose si la patiente refuse les soins. Donc l'idée c'est que la patiente et le soignant se déplacent un petit peu pour atteindre un objectif de soins commun. Mieux vaut un traitement un tout petit peu moins efficace mais bien accepté par la patiente qu'un traitement un tout petit peu plus efficace, rejeté [...] C'est pour cela qu'on doit être côte à côte : nous sommes deux à nous engager. »

Cette esquisse d'horizontalisation des interactions de soins façonnent non seulement les positions spatiales mais également les rôles et les places genrées, relatives aux soignant.e.s « savant.e.s » et aux malades «profanes ». Si l'ergonomie de consultations est ici un indice du souci du genre, nous ne pouvons toutefois avancer le même constat avec des patient.e.s atteint.e.s d'autres types de cancer considérés dans le cadre de la présente enquête (prostate, poumon, colorectal), ce qui nous incite à limiter la valeur hermé-neutique de cette observation au stade d'hypothèse.

Conclusion

$\mathrm{Si}$ notre travail se focalise sur la place du genre dans les inter-actions soignant.e.s/soigné.e.s en cancérologie, il n'en demeure pas moins une porte d'entrée plus générale à l'observation des phénomènes en lien avec le genre en œuvre dans les parcours de soins dans leur ensemble. Dans ce sens, cette perspective inaugure une nouvelle piste interprétative des relations soignant.e.s/ soigné.e.s souvent observées 
exclusivement du côté des patient.e.s elles/eux-mêmes. Or l'analyse montre la nécessité de s'intéresser aux différents acteurs en jeux et d'appliquer une approche

intersectionnelle, afin de saisir ce traitement différencié des femmes et des hommes qui, en contexte des soins, ne se laisse pas facilement appréhender.

L'analyse montre que si certain.e.s praticien.ne.s observé.e.s s'adaptent et modifient leurs pratiques au contact de nouveaux regards qui introduisent le genre, d'autres restent sceptiques. Mais, généralement, la prise en compte des questions de genre en régime de santé ne relève pas de l'évidence. Toutefois, si l'on considère qu'une médecine non maltraitante est une médecine qui prend en considération les caractéristiques singulières des corps des malades (qu'il s'agisse des femmes, des hommes ou des minorités de genre et de sexualité), alors une approche sociologique des subjectivités genrées est indispensable afin de lever les résistances qui s'y déploient, et de proposer des soins plus adaptés au profil de la patientèle.

Enfin, inoculer le genre dans les études de santé et plus particulièrement dans les analyses relatives aux interactions soignant.e.s/soigné.e.s permet de redimensionner, sans pour autant remettre en cause, ce qui a été souvent analysé du côté de l'oubli et de la domination des femmes dans les soins (Salle, Vidal, 2017; Membrado, 2006). Comme le soulignent les regards microsociologiques sur les interactions de soins et les interpré-tations en termes de croyances en régime de santé (Hammer, 2010), une analyse des rapports interindividuels dans la santé doit compter sur les notions de confiance et de loyauté. Nous faisons alors le constat que les identités et les expressions de genre des patient.e.s comme des soignant.e.s s'entremêlent à ces émotions, dans un contexte de santé sous tension où les identités de genre des malades demeurent encore largement impensées.

Bibliographie

Abelhauser, A. ; Levy, A. ; Laska, F. ; Weil-Philippe, S. 2001. «Le temps de 1'adhésion », dans anrs, L'observance aux traitements contre le vih-sida. Mesures, déterminants, évolutions, Collection Sciences sociales et Sida, p. 79-86.

Ahmed, R.; Bates, B. 2007. «Gender differences in patients'perceptions of physicians'cultural competence in health care interactions ", Women's Health \& Urban Life, vol. $6, \mathrm{n}^{\circ} 2$, p. 58-80. 
Alessandrin, A. (2018), Sociologie des transidentités, Cavalier Bleu.

Alessandrin, A. ; Dagorn, J. ; Meidani, A. ; Richard, G. ; Toulze, M. (2020). Santé LGBT, Bord de l'eau.

Alessandrin, A. ; Esteve-Bellebeau, B. 2014. Genre! L'essentiel pour comprendre, Paris, Des ailes sur un tracteur.

Alessandrin, A.; Meidani, A. 2018a. «Cancer(s), questions de genre et identités sexuelles », Santé scolaire et universitaire, $\mathrm{n}^{\circ} 51$ (sous la direction de E. Dugas), p. 16-20.

Alessandrin, A. ; Meidani, A. 2018b. «Corps, cancers et genre », Pratiques, n 80, p. 71-72.

Alessandrin, A. ; Toulze, M, (2020), « Minorités sexuelles et rapport au corps : une recherche quantitative », Santé Publique, vol. 31, no. 5, pp. 657-661.

Ankeny, R.-A. 2014. "Maladie et santé : concepts et représentations », dans B. Fantini, L. Lambrichs (sous la direction de), Histoire de la pensée médicale contemporaine: évolutions, découvertes, controverses, Paris, Le Seuil, p. 11-24.

Arapoglou, V.-P. 2004. «The governance of homelessness in the Euro-pean South: Spatial and institutional contexts of philanthropy in Athens », Urban Studies, vol. 41, n 3, p. 621 639.

Aronowitz, R.-A. 2001. «Do not delay: Breast cancer and time 1900-1970», The Milbank Quarterly, vol. 79, n³, p. 355-386.

Aronowitz, R.-A. 2007. Unnatural History: Breast Cancer and American Society, Cambridge / New York, Cambridge University Press.

Arrington, M.-I. 2004. «To heal or not to heal ?: On prostate cancer, physician-patient communication and sexuality », Journal of Loss and Trauma, vol. 9, n² 2, p. 159-166.

Arrington, M.-I. 2008. «Prostate cancer and the social construction of masculine sexual identity », International Journal of Men's Heath, vol. 7, n³, p. 299-306.

Avenel, C. 2012. «La question de l'égalité des parcours en faculté de médecine: les conditions d'entrée à l'université des nouvelles cara-bines », Education et socialisation, $\mathrm{n}^{\circ} 31$, [En ligne : https://journals. openedition.org/edso/801].

Bajos, N.; Bozon, M. (sous la direction de). 2008. Enquête sur la sexua-lité en France. Pratiques, genre et santé, Paris, La Découverte.

Bajos, N.; Bozon, M. 2011. «Les transformations de la vie sexuelle après 50 ans: un vieillissement genré $»$, Genre, sexualité et société, $\mathrm{n}^{\circ} 6$ [en ligne : https://gss.revues.org/2165].

Barbot, J. 2002. Les malades en mouvements. La médecine et la science à l'épreuve du sida, Paris, Balland. 
Barnay, T. 2005. "Santé déclarée et cessation d'activité », Revue fran-çaise d'économie, vol. $20, \mathrm{n}^{\circ} 2$, p. 351 73- 106. [En ligne : https://doi. org/10.3406/rfeco.2005.1573].

Barnay, T. 2008. "Chômage et invalidité après 50 ans : deux disposi-tifs alternatifs de sortie de l'emploi pour les seniors en mauvaise santé ? », Économie et statistique, $\mathrm{n}^{\circ} 411$, p. 47-63.

Barnay, T.; Sauze, D. ; Sultan-Taïeb, H. 2010. «La santé au travail : une préoccupation multiforme pour les économistes », Revue française des affaires sociales, $n^{\circ} 4$, p. 7-25.

Barthe, J.-F. ; Clément, S. ; Drulhe, M. 1988. «Vieillesse ou vieillis-sement? Les processus d'organisation des modes de vie chez les personnes âgées », Les cahiers de la recherche sur le travail social, $\mathrm{n}^{\circ} 15, \mathrm{p} .11-31$.

Bataille, P. 2003. Un cancer et la vie. Les malades face à la maladie, Paris, Balland.

Bauer, G.-R. ; Scheim, A.-I. ; Deutsch, M.-B. ; Massarella, C. 2014. « Reported emergency department avoidance, use, and expe-riences of transgender persons in Ontario, Canada: Results from a respondent-driven sampling survey », Annals of Emergency Medicine, vol. $63, \mathrm{n}^{\circ} 6$, p. $713-720$.

Beauvoir, S. de. 1949. Le deuxième sexe, Paris, Gallimard, 1986.

Ben Halima, B. ; Ben Halima, M.-A. ; Lanfranchi, J. ; Le Clainche, C. ; Regaert, C. 2016. L'impact du diagnostic du cancer sur les transitions professionnelles en France : une étude sur données de panel administra-tives, Paris, Noisy-le-Grand, Centre d'études de l'emploi et du travail

Bentz, E.-K.; Pils, D.; Bilban, M. ; Kaufmann, U. et coll. 2010. "Gene expression signatures of breast tissue before and after cross-sex hormone therapy in female-to-male transsexuals », Fertility and Sterility, vol. 94, n 7, p. 2688-2696.

Berrino, F.; Cascinelli, N. 1999. «Cancer prevalence. What for?», Tumori, vol. 85, p. 414-447.

Bize, R. ; Volkmar, E. ; Berrut, S. ; Medico, D. ; Balthasar, H. ; Bodenmann, B. ; Makadon, H.-J. 2011. «Vers un accès à des soins de qualité pour les personnes lesbiennes, gays, bisexuelles et trans-genres », Revue médicale suisse, $n^{\circ}$ 7, p. 1712-1717.

Blank, T. 2005. «Gay men and prostate cancer: Invisible diversity », Journal of Clinical Oncology, vol. 23, n 12, p. 2593-2596.

Blashill, A. ; Pagoto, S. 2015. «Skin cancer risk in gay and bisexual population », Jama Dermathology, vol. 151, ${ }^{\circ} 12$, p. 1293-1325.

Boehmer, U. 2011. «Cancer survivorship and sexual orientation », Cancer, vol. 117, $\mathrm{n}^{\circ} 16$, p. 3796-3804.

Boehmer, U.; Elk, R. 2015. Cancer and the lgbt Community: Unique Perspectives from Risk to Survivorship, Berlin, Springer.

Boehmer, U. 2018. «lgbt populations' barriers to cancer care », Onco-logy Nursery, vol. $34, \mathrm{n}^{\circ} 1$, p. 21-29.

Boehmer, U. ; Glickman, M. ; Winter, M. ; Clark, M.-A. 2013. « Long-term breast cancer survivors' symptoms and morbidity: Differences by sexual orientation? », Journal of Cancer Survivorship, vol. 7, n², p. 203-210.

Boltanski, L. 1971. «Les usages sociaux du corps », Annales esc, vol. 26, n 1, p. 205-233. 
Bondil, P.; Habold, D. 2015. «Développement de l'oncosexualité et oncofertilité en France : aspects culturels et psychosociologiques "), Psycho-oncologie, n 9, p. 230-239.

Bondil, P. ; Habold, D. ; Damiano, D. ; Champsavoir, P. 2012. «Le parcours personnalisé de soins en oncosexologie: une nouvelle offre de soins au service des soignés et des soignants », Bulletin du cancer, n 99, p. 499-507.

Bonnet, C. ; Hourriez, J.-M. 2012. «Égalité entre hommes et femmes à la retraite : quels rôles pour les droits familiaux et conju-gaux? », Population, vol. 67, n 1, p. 133- 158 [en ligne : https://doi. org/10.3917/popu.1201.0133].

Bontron, J.-C. 2012, «Les pôles d'excellence rurale, laboratoires des nouveaux rapports santé-territoires », Pour, vol. 2, n 214, p. 113-119.

Boorse, C. 1977. «Health as a theoretical concept», Philosophy of Science, $n^{\circ} 44$, p. 542 573.

Bouchut, E. 1853. Des méthodes de classification en nosologie, Paris, Martinet.

Bouhnik, A.-D.; Bendiane, M.-K.; Cortaredona, S.; Sagaon Teys-sier, L.; Rey, D. ; Berenger, C. ; Seror, V. et coll. 2015. «The labour market, psychosocial outcomes and health conditions in cancer survivors: Protocol for a nationwide longitudinal survey 2 and 5 years after cancer diagnosis (the vican Survey) », bmj Open, vol. 5, $n^{\circ} 3$, art. $n^{\circ}$ e005971 [9 p. en ligne : https://journals.openedition. org/efg/1346].

Bourdieu, P. 2002. La domination masculine, Paris, Le Seuil.

Bourgeois, I. 2015. "Les dimensions cognitives de l'intervention en santé publique: l'accompagnement de deux projets de santé de premier recours en milieu rural», Santé publique, vol. $27, \mathrm{n}^{\circ} 3$, p. 343-351.

Braverman, L. 2017. «Masculinités vieillissantes à l'épreuve du cancer de la prostate», Enfances familles générations, $n^{\circ} 27$, [en ligne : https://journals.openedition.org/efg/1346].

Braverman, L. 2017a. «"Il n’y a jamais rien qui est entré par-là !'” Résis-tances et malaises masculins face au toucher rectal », Recherches sociologiques et anthropologiques, vol. 48, $\mathrm{n}^{\circ} 1, \mathrm{p} .45-64$.

Braverman, L., 2018, «Le sujet à risque : la mesure du psa dans l'ex-périence du cancer de la prostate ", dans N. Amsellem et P. Bataille (sous la direction de), Cancer, un regard sociologique. Biomédicalisa-tion et parcours de soins, Paris, La Découverte, p. 15-27.

Briot, M. 2006. Rapport parlementaire, $\mathrm{n}^{\circ} 3125$ [en ligne: http://www.assembleenationale.fr/12/rap-off/i3187.asp].

Broom, A. 2004. «Prostate cancer and masculinity in australian society: A case of stolen identity? », International Journal of Men’s Health, vol. 3, n² 2, p. 73-91.

Broom, A. 2012. «Gender and end-of-life care », dans E. Kuhlmann et E. Annandale (sous la direction de), The Palgrave Handbook of Gender and Healthcare, Londres, Palgrave Macmillan, p. 224-238.

Broom, A.; Cavenagh, J. 2010. «Moralities, masculinities and caring for the dying», Social Science and Medicine, vol. 71, $\mathrm{n}^{\circ}$ 5, p. 869-876.

Brugère, F. 2017. L'éthique du care, Paris, Puf.

Brullmann, F., 2007. «Du traumatisme de l'ablation d'un sein après cancer à la reconstruction réparatrice : une traversée », Le Carnet Psy, n 119, p. 46-51. 
Bryson, M.-K.; Stacey, J. 2013. «Cancer knowledge in the plural: Quee-ring the biopolitics of narrative and affective mobilities », Journal of Medical Humanities, vol. 34, $\mathrm{n}^{\circ} 2$, p. 197-212.

Bryson, M.-K. ; Taylor, E. ; Boschman, L. ; Hart, T.; Gahagan, J. ; Rail, G. ; Ristock, J. 2018. "Awkward choreographies from Cancer's Margins: Sexual and/or gender minority cancer patients' encounters with biographical and biomedical knowledge in treatment », Journal of Medical Humanities, vol. 7, $\mathrm{n}^{\circ} 1$, p. 102-113 [en ligne: https://doi. org/10.1007/s10912-018-9542-0].

Buller, M. ; Buller, D. 1987. «Physicians' communication style and patient satisfaction », Journal of Health and Social Behavior, $n^{\circ} 28$, p. 375-388.

Bureau, E. ; Hermann-Mesfen, J. 2014. «Les patients contemporains face à la démocratie sanitaire $», \quad$ Anthropologie \& Santé, $\mathrm{n}^{\circ} 8$ [en ligne : https://journals.openedition.org/anthropologiesante/1342].

Burguière, A. 1988. «L'anthropologie historique », dans J. Le Goff (sous la direction de), La nouvelle histoire, Bruxelles, Complexes, p. 137-165.

Burkhalter, J. 2010. «Perceived risk for cancer in an urban sexual minority », International Journal of Behavior Medicine, vol. 34, n³, p. 157-169.

Burkhalter, J.-E. ; Margolies, L.; Sigurdsson, H.-O.; Walland, J. ; Radix, A. ; Rice, D. ; Maingi, S. 2016. «The national lgbt cancer action plan: A white paper of the 2014 national summit on cancer in the lgbt Communities », lgbt Health, vol. 3, n 1, p. 19-31.

Bury, M. 1982. «Chronic illness as a biographical disruption », Sociology of Health and Illness, vol. 4, n², p. 167-182.

Butler, J. 2005. Humain, inhumain, le travail critique des normes, Paris, Éditions Amsterdam.

Butler, J. 2006. Trouble dans le genre. Le féminisme et la subversion de l'identité [trad. de l'américain par C. Kraus], Paris, La Découverte.

Butler, J. 2007. Le récit de soi, Paris, Puf (titre en anglais : Giving an account of oneself, trad. par B. Ambroise et V. Aucouturier).

Bylund, C.; Makoul, G. 2002. «Empathic communication and gender in the physicianpatient encounter $»$, Patient Education and Counse-ling, $n^{\circ} 48$, p. 207-216.

Cahill, S. 2018. «Legal and policy issues for lgbt patients with cancer or at elevated risk of cancer $»$, Oncology Nursery, vol. 34, n 1, p. 90-98.

Calvez, M. 2004. La prévention du sida. Les sciences sociales et la définition des risques, Presses universitaires de Rennes.

Calvez, M. ; Paicheler, G. ; Souteyrand, Y. (sous la direction de). 1994. Connaissances, représentations, comportements: les sciences sociales et la prévention du sida, Paris, Agence nationale de recherche sur le sida.

Canguilhem, G. 1966. Le normal et le pathologique, Paris, Puf.

Canguilhem, G. 2002. Écrits sur la médecine, Paris, Le Seuil. 
Carby, H. 2007. «Femme blanche écoute! Le féminisme noir et les frontières de la sororité », dans E. Dorlin (sous la direction de), Black Feminism. Anthologie du féminisme africain-américain, Paris, L'Harmattan.

Carricaburu, D.; Ménoret, M. 2004. Sociologie de la santé. Institutions, professions et maladies, Paris, Armand Colin.

Casper, M.-J.; Clarke, A.-E. 1998. «Making Pap smear into the 'right tool' for the job: Cervical cancer screening in the usa, circa 1940-1995», Social Studies of Science, vol. 28, $\mathrm{n}^{\circ} 2$, p. $255-290$.

Cathcart-Rake, E. 2018. «Cancer in sexual and gender minority patients: Are we addressing their needs? », Current Oncology Reports, vol. 20, n 11, p. 18.

Chapple, A. ; Ziebland, S. 2002. «Prostate cancer: Embodied experience and perceptions of masculinity », Sociology of Health and Illness, vol. 24, n 6 , p. 820-841.

Charif, A.; Bouhnik, A.-D. ; Rey, D. ; Mancini, J. 2015. « Satisfaction with fertility and sexuality-related information in young women with breast cancer-elippse40 Cohort », bmc Cancer, vol. $15, \mathrm{n}^{\circ} 1$, p. 572-583.

Chartrain, C. 2009. «Pour une promotion de la santé lesbienne. État des lieux des recherches, enjeux et propositions », Genre, sexualité et société, $n^{\circ} 1$, [en ligne : https://journals.openedition.org/gss/951].

Chartrain, C. (sous la direction de). 2013. «Les jeunes face aux discri-minations liées à l'orientation sexuelle et au genre : agir contre les lgbt-phobies », Cahiers de l'action, $\mathrm{n}^{\circ} 40$.

Chassain, A. ; Clochec, P. ; Le Meur, C. ; Lenormand, M. ; Trégan, M. 2016. «Approches expérientielles du fait minoritaire », Tracés. Revue de sciences humaines, $\mathrm{n}^{\circ}$ 30, p. 7-26, [en ligne : https://jour-nals.openedition.org/traces/6397\#quotation].

Clark, L. ; Holcombe, C. ; Hill, J. ; Krespi-Boothby, M.-R. ; Fisher, J. ; Seward, J. ; Salmon P. 2011. "Sexual abuse in childhood and post- operative depression in women with breast cancer who opt for immediate reconstruction after mastectomy », Annals of the Royal College of Surgeons of England, vol. 93, n² 2, p. 106-10.

Clarke, A.-E. ; Shim, J.-K. ; Mamo, L. ; Fosket, J.-R. ; Fishman, J.-R., 2003, «Biomedicalization: Technoscientific transformations of health, illness, and US biomedicine ", American Sociological Review, vol. 68, n² 2, p. 161-194.

Cognet, M. 2017, «Du traitement différentiel à la discrimination raciste dans les pratiques cliniques », Les cahiers de la lcd, vol. 3, n 5, p. 25-42.

Cohen, P.; Sarradon-Eck, A. ; Rossi, I. ; Schmitz, O. ; Legrand, É. 2015. Cancer et pluralisme thérapeutique: enquête auprès des malades et des institutions médicales en France, Belgique et Suisse, Paris, L'Harmattan.

Collectif de Boston pour la santé des femmes. 1977. Notre corps nous-mêmes, Paris, Albin Michel.

Connell, R.-W. 1987. Gender and Power: Society, the Person and Sexual Politics, Londres, Polity.

Connell, R.-W. 2002. «On hegemonic masculinity and violence: Response to Jefferson and Hall », Theoretical Criminology, vol. 6, n 1, p. 89-99.

Connell, R.-W. 2005 [1995]. Masculinities, Berkeley, University of Cali-fornia Press.

Connell, R.-W. 2014. Masculinités. Enjeux sociaux de l'hégémonie, Paris, Amsterdam. 
Corbin, A. 1982. Le miasme et la jonquille. L'odorat et l'imaginaire social (xviiiexixe siècles), Paris, Aubier Montaigne.

Corbin, J.; Strauss, A. 1988. Unending Work and Care: Managing Chronic Illness at Home, San Francisco, Jossey-Bass.

Courtenay, W. 2000. «Constructions of masculinity and their influence on well-being: A theory of gender and health », Social Science \& Medicine, vol. 50, n 10, p. 1385-1401.

Crenshaw, K. 1991. "Mapping the margins: Intersectionality, iden-tity politics, and violence against women of color », Stanford Law Review, vol. 43, n 6, p. 1241-1299.

Cresson, G. 2001. Le travail sanitaire profane dans la famille : analyse sociologique, thèse pour l'habilitation à diriger des recherches en sociologie, Paris, ehess.

Cresson, G. ; Gadrey, N. 2004. «Entre famille et métier: le travail du care », Nouvelles questions féministes, vol. $23, \mathrm{n}^{\circ} 3$, p. 26-41.

Cunin, E. 2001. «La compétence métisse. Chicago sous les tropiques ou les vertus heuristiques du métissage », Sociétés contemporaines, $n^{\circ} 42$, p. 7-24.

Dagorn, J. ; Alessandrin, A. 2015. «Être une fille, un gay, une lesbienne ou un.e trans au collège et au lycée », Le sujet dans la cité, vol. 6, n² 2, p. 140-149.

Dagorn, J.; Alessandrin, A. 2018. «La santé des lgbti à l'école», L'école des parents, $\mathrm{n}^{\circ}$ 627, p. 43-46.

Dale, W.; Sartor, S.; Davis, T.; Bennett, C.-L. 1999. «Understan-ding barriers to the elderly detection of prostate cancer among men of lower socioeconomic status », Prostate Journal, vol. 1, n 4, p. 176-184.

Damaskos, P. ; Amaya, B. ; Gordon, R. ; Walters, C. 2018. «Intersec-tionality and the lgbt cancer patient », Oncology Nursery, vol. 34, n 1, p. 30-36.

Dares. 2015. «Ségrégation professionnelle et écarts de salaires femmes-hommes », $\mathrm{n}^{\circ} 82$ [en ligne : https://ares.travail-emploi.gouv.fr/ IMG/pdf/2015-082.pdf].

Da Silva, P. 2013. Histoire de la recherche de substances naturelles à acti-vités thérapeutiques, thèse d'épistémologie et histoire des sciences et des techniques, université Bordeaux 1.

Dayer, C. 2014. «Transcolarité », dans K. Espineira, M.-Y. Thomas et A. Alessandrin (sous la direction de), Tableau noir : les transidentités et l'école, Paris, L'Harmattan, p. 13-17.

Dayer, C. 2016. «Socialisation en négatif: des situations de vulnérabilité aux conditions d'apprentissage », Tréma-Revue internationale en sciences de l'éducation et didactique, $\mathrm{n}^{\circ} 46$, p. 51-61.

Dayer, C. 2017. Le pouvoir de l'injure. Guide de prévention des violences et des discriminations, La Tour d'Aigues, Éditions de l'Aube.

Derbez, B. ; Rollin, Z. 2016. Sociologie du cancer, Paris, La Découverte. Delphy, C. 2001. L'ennemi principal. 2. Penser le genre, Paris, Syllepse. Delpierre, C. ; Lamy, S. ; KellyIrving, M. ; Molinié, F. ; Velten, M. ;

Tretarre, B. ; Woronoff, A.-S. ; Buemi, A. ; Lapôtre-Ledoux,

B.; Bara, S.; Guizard, A-V.; Colonna, M.; Grosclaude, P. 2013. «Life expectancy estimates as a key factor in over-treatment: The case of prostate cancer », Cancer Epidemiology, vol. 37, ${ }^{\circ}$ 4, p. 462-468. 
Demazeux, S. 2013. Qu'est-ce que le dsm? Genèse et transformations de la bible américaine de la psychiatrie, Paris, Ithaque.

Diedrich, L. 2007. Treatments, Minneapolis, University of Minnesota Press.

Dorlin, E. ; Fassin, E. (sous la direction de). 2010. Reproduire le genre, Paris, Bibliothèque publique d'information du Centre Pompidou.

Douglas, M. 1966. Purity and Danger: An Analysis of Concepts of Pollu-tion and Taboo, Londres, Routledge.

Dowsett, G.-W. 2008. "'Losing my chestnut': One gay man's wrangle with prostate cancer », Reproductive Health Matters, vol. 16, n 32, p. 145-150.

Dresher, J. 2010. «Queer diagnoses: Parallels and contrasts in the history of homosexuality, gender variance, and the diagnostic and statistical manual (dsm) », Archives of Sexual Behavior, $n^{\circ} 39$, p. 427-460.

Dubet, F. 2001. Sociologie de l'expérience, Paris, Le Seuil.

Dubet, F. 2017. L’expérience sociologique, Paris, La Découverte.

Duffy, M. 2005. «Reproducing labor inequalities: Challenges for femi-nists conceptualizing care at the intersections of gender, race, and class », Gender \& Society, vol. $19, \mathrm{n}^{\circ} 1$, p. 66-82.

Duguet, E.; Le Clainche, C. 2016. «Une évaluation de l'impact de l'aménagement des conditions de travail sur la reprise du travail après un cancer », Revue économique, vol. 67, $\mathrm{n}^{\circ} 1$, p. 49- 80 [en ligne : https://doi.org/10.3917/reco.pr2.0053. 372].

Dziengel, L. 2014. «Renaming, reclaiming, renewing the self: Intersec-tions of gender, identity, and health care », Affilia: Journal of Women and Social Work, vol. 29, $\mathrm{n}^{\circ} 1$, p. $105-110$.

Edelman, N. 2003. «Discours médical et construction des catégories homme/femme, masculin/féminin », Revue internationale, $\mathrm{n}^{\circ} 6$ [en ligne: 2003/06-http:/www.senspublic.org/spip.php ?article9].

Edelman, N. 2003. Les métamorphises de l'hystérique. Du début du xixe siècle à la Grande Guerre, Paris, La Découverte.

Ehrenberg, A. 2005. "Agir de soi-même », Esprit, p. 200-209.

Eribon, D. 1999. Reflexions sur la question gay, Paris, Flammarion.

Eribon, D. (sous la direction de). 2003. Dictionnaire des cultures gays et lesbiennes, Paris, Larousse.

Fassin, D. 1996. L’espace politique de la santé. Essai de généalogie, Paris, Puf.

Fassin, D. 1998. «Les politiques de la médicalisation », dans P. Aïach et D. Delanoë, (sous la direction de), L'ère de la médicalisation. Ecce homo sanitas, Paris, Economica, p. 1-13.

Fassin, D. 2000a. «Politiques de la vie et politiques du vivant. Pour une anthropologie de la santé », Anthropologie et sociétés, numéro spécial « Terrains d'avenir », vol. 24, n 1, p. 95 116.

Fassin, D. 2000b. «Repenser les enjeux de santé autour de l'immigra-tion », Hommes et migrations, $\mathrm{n}^{\circ} 1225$, p. 5-12. 
Fassin, D. 2000c. «Le culturalisme pratique de la santé publique. Critique d'un sens commun ", dans J.-P. Dozon et D. Fassin (sous la direction de), Critique de la santé publique, Paris, Balland, p. 181-208.

Fassin, D. 2010. Sexe, race, classe, pour une épistémologie de la domination, Paris, Puf.

Fassin, D. 2011. Sexe, genre et sexualités, Paris, Puf.

Faulkner, A. 2010. «Trial, trial, trial again: Reconstructing the gold stan-dard in the science of prostate cancer detection », dans C. Will et T. Moreira (sous la direction de), Medical Proof/Social Expériments: Clinical Trials in Shifting Contexts, Farnham, Ashgate, p. 137152.

Fay, C. 1999. «Du "culturel", de 1" "universel" ou du "social"? Penser le sida et la prévention au Mali », dans C. Becker, J.-P. Dozon et coll. (sous la direction de), Vivre et penser le sida en Afrique [Experiencing and Understanding Aids in Africa], Paris, Codesria-Karthala-ird, p. 277-298.

Fentiman, I. 2017. Male Breast Cancer, New York, Springer International. FerrandBechmann, D. 2011. Les bénévoles face au cancer, Bruxelles,

Desclée de Brouwer.

Filiault, S. ; Drummond, M.; Smith A. 2008. «Gay men and prostate cancer: Voicing the concerns of a hidden population », Journal of Men's Health, vol. 5, n 4, p. 327-332.

Fobair, P. ; O’Hanlan, K. ; Koopman, C. ; Classen, C. ; Dimiceli, S. ; Drooker, N. ; Warner, D. et coll. 2001. "Comparison of lesbian and heterosexual women's response to newly diagnosed breast cancer », Psycho-Oncology, vol. 10, n 1, p. 40-51.

Foss, C. 2002. "Gender bias in nursing care? Gender-related differences in patient satisfaction with the quality of nursing care », Scandina-vian Journal of Caring, $\mathrm{n}^{\circ} 16$, p. 19-26.

Frazer, J.-G. 1984. Les rameaux d'or, Paris, Robert Laffont.

Freidson, E. 1984. La profession médicale, Paris, Payot.

Gadamer, H-G. 1998. Philosophie de la santé, Paris, Grasset-Mollat.

Gallioz, S. 2006. "Force physique et féminisation des métiers du bâti-ment», Travail, genre et sociétés, $\mathrm{n}^{\circ} 16$, p. 97- 114 [en ligne : https:// doi.org/10.3917/tgs.016.0097.374].

Gardey, D. ; Löwy, I. 2000. L'invention du naturel: les sciences et la fabrication du féminin et du masculin, Paris, Éditions des archives contemporaines.

Gardner, K.-E. 2006. Early Detection: Women, Cancer, and Awareness Campaigns in the Twentieth-Century United States, Chapel Hill, University of North Carolina Press.

Giami, A. 2004. «De l'impuissance à la dysfonction érectile. Destins de la médicalisation de la sexualité », dans D. Fassin et D. Memmi (sous la direction de), Le gouvernement des corps, Éditions de l'École des hautes études en sciences sociales, p. 77-108.

Gibbon, S. 2007. Breast Cancer Genes and the Gendering of Knowledge: Science and Citizenship in the Cultural Context of the 'New' Genetics, Londres, Palgrave Macmillan.

Gibbon, S. ; Carlos, N. (sous la direction de). 2008. Biosocialities, Gene-tics and the Social Sciences: Making Biologies and Identities, Londres, Routledge. 
Gilligan, C. 2008. Une voix différente, Paris, Flammarion.

Girard, G. 2013. Les homosexuels et le risque du sida. Individu, commu-nauté et prévention, Presses universitaires de Rennes.

Girard, G. ; Bergoudo, F. 2017. La fin du sida est-elle possible ?, Patris, Textuel.

Giraud, M. 1979. Races et classes à la Martinique, Paris, Anthropos.

Giroux, É. 2009. «Définir objectivement la santé : une évaluation du concept biostatistique de Boorse à partir de l'épidémiologie moderne », Revue philosophique de la France et de l'étranger, $\mathrm{n}^{\circ} 1$ (tome 134), p. 35-58.

Godelier, M. 1982. La production des grands hommes, Paris, Fayard.

Gordon, D.-R.; Eugenio P. 1997. «Disclosure practices and cultural narratives: Understanding concealment and silence around cancer in Tuscany, Italy », Social Science and Medicine, vol. 44, $\mathrm{n}^{\circ}$ 10, p. 1433-1452.

Gori, R. ; Del Volgo, M.-J. 2004. La santé totalitaire, Paris, Denoël.

Gray, R.; Fitch, M.; Fergus, K.; Mykhalovkiy, E.; Church, K. 2002. «Hegemonic masculinity and the experience of prostate cancer: Narrative approach », Journal of Aging and Identity, $\mathrm{n}^{\circ} 7$, p. 43-62.

Gray, R. ; Fitch, M. ; Phillips, C. ; Labrecque, M. ; Fergus, K. 2000. « To tell or not to tell: Paterns of disclosure among men with pros-tate cancer $»$, Psycho-Oncology, $n^{\circ}$ 9, p. 273282.

Greco, C. 2016a. « Shining a light on the grey zones of gender construc-tion: Breast surgery in France and Italy », Journal of Gender Studies, vol. 25, n 3, p. 303-317.

Greco, C. 2016b. «Vivre avec un corps asymétrique. Mastectomie, résis-tances et réappropriation », Cahiers du genre, $n^{\circ} 60$, p. 81-99.

Green B. B. ; Taplin S.-H. 2003. «Breast cancer screening controver-sies », Journal of the American Board of Family Medicine, vol. 16, n 3, p. 233-241.

Grindel, C.-G. ; Mcgehee, L. ; Patsdaughter, C. ; Roberts, S. 2006. « Cancer prevention and screening behaviors in lesbians », Women and Health, vol. 44, $\mathrm{n}^{\circ} 2$, p. 15-39.

Grmek, M.D. 1969. «Préliminaires d'une étude historique des mala-dies », Annales esc, vol. 24, n 6 , p. 1473-1483.

Grmek, M.D. 1999-2014. «Le concept de maladie », dans Histoire de la pensée médicale en Occident; vol.1: Antiquité et Moyen Âge, 2014; vol. 2: De la Renaissance aux Lumières, 2014 ; vol. 3 ; Du romantisme à la science moderne 1999.

Gros, D. 1994. Les seins aux fleurs rouges, Paris, Stock.

Gros, D. 2009. Cancer du sein, entre raison et sentiments, Paris, Springer Verlag France.

Guillaumin, C. 1992. Sexe, race et pratique du pouvoir : l'idée de nature, Paris, Indigo \& Côté-femmes.

Guthrie, C. 2018. Flat: Reclaiming my Body from Breast Cancer, New York, Skyhorse Publishing.

Hackshaw, A.-K. ; Paul E.-A. 2003. « Breast self examination and death from breast cancer: A meta-analysis », British Journal of Cancer, vol. 88, n 7, p. 1047-1053. 
Hall, L. 1997. «Re-figuring marked bodies on the borders: Breast cancer and 'femininity' ", International Journal of Sexuality and Gender Studies, vol. 2, $\mathrm{n}^{\circ} 2$, p. 101 21.

Hammer, R. 2010. Expériences ordinaires de la médecine: confiances, croyances et critiques profanes, Zurich, Éditions Seismo

Heck, J. et coll. 2010. «Sexual behaviours and the risk of head and neck cancers: A pooled analysis », Int J. Epidemiol, n 39, p. 166-181.

Héquet, D. et coll. 2013. «Reasons of not having breast reconstruc-tion: A historical cohort of 1937 breast cancer patients undergoing mastectomy », SpringerPlus, n² 2, p. 325 [en ligne].

Herzlich, C. 1995. «Les difficultés de constitution d'une cause », Sciences sociales et santé, vol. $13, n^{\circ} 4$, p. 39-44.

Herzlich, C. 2005. Santé et maladie. Analyse d'une représentation sociale, Paris, École des hautes études en sciences sociales.

Herzlich, C. ; Pierret, J. 1984. Malade d'hier, malade d'aujourd'hui. Paris, Payot.

Hiestand, K.-R.; Horne, S.-G. ; Levitt, H.-M. 2007. «Effects of gender identity on experiences of healthcare for sexual minority women », Journal of lgbt Health Research, vol. $3, n^{\circ} 4$, p. 15-27.

Hirschon, R. 2007. «Imported individuals and indigenous persons: Changing paradigms of personal identity in contemporary Greece », Ethnologia, n 13, p. 217-237.

Hœrni, B. (sous la direction de). 2006. Dictionnaire des cancers. Histoire, Science, Médecine, Société, Paris, Éditions Frison-Roche.

Horgues-Debat, J. 2009. «Le rôle des associations pour le dévelop-pement des services à la population en milieu rural », Pour, vol. 2, n 201, p. 149-153.

Howyan Gros, C.-J. 1989. Le traitement psychologique dans la maladie cancéreuse, thèse de sciences de l'éducation, Paris, université Paris VIII.

Hunt, K. ; Macintyre, S. 2000. "Genre et inégalités sociales en santé », dans D. Fassin (sous la direction de), Les inégalités sociales de santé, Paris, La Découverte, p. 363- 375 [en ligne: https://www.cairn.info/ les-inegalites-sociales-de-sante--9782707132475-page363.htm].

INCa. 2014. La vie deux ans après un diagnostic de cancer. De l'annonce à l'après cancer, Paris, inca [en ligne].

INCa. 2018. La vie cinq ans après un diagnostic de cancer, Paris, inca [en ligne].

Institute Of Medicine. 2011. The Health of Lesbian, Gay, Bisexual, and Transgender People: Building a Foundation for Better Understanding, National Academies Press, Washington.

Jedrzejewki, T. (sous la direction de). 2016. Enquête gays et lesbiennes. Médecine générale, Paris, inpes.

Johnstone E.-B. ; Baines, J. 2015. The Changing Faces of Childhood Cancer: Clinical and Cultural Visions Since 1940, Basingstoke, Palgrave Macmillan.

Joynt, C ; Bryson, M.-K. 2013. «Under the skin; Imag(in)ing medicines queer pedagogies as moving pictures », No More Potlucks, Fall. 
Joynt, C.; Mary, B. 2012. Resisterectomy, consulté le 27 septembre, 2018 [en ligne: https://www.chasejoynt.com/resisterectomy].

Kamen, C. ; Smith-Stoner, M. ; Heckler, C. ; Flannery, M. ; -Margolies, L. 2015. «Social support, self-rated health, and lesbian, gay, bisexual, and transgender identity disclosure to cancer care providers », Oncology Nursing Forum, vol. 42, n 1, p. 44-51.

Kampriani, E. 2009. «Between religious philanthropy and individualised medicine: Situating inherited breast cancer risk », Greece Anthropo-logy \& Medicine, vol. 16, $\mathrm{n}^{\circ} 2$, p. $165-178$.

Karakasidou, A. 2007. "The bio-politics of cancer: Experience, social metaphor and authoritative knowledge », Archeologia \& Technes, $n^{\circ} 105$, p. 55-62.

Kenerson, D. 2010. Use of the Theory of Planned Behavior to Assess Pros-tate Cancer Screening Intent among African American Men, thèse Vanderbilt University.

King, S. 2006. Pink Ribbons, Inc. Breast Cancer and the Politics of Philantropy, Minneapolis, University of Minnesota Press.

Kivits, J. ; Fournier, C. ; Mino, J.-C. et coll. 2013. «Jalons pour une recherche interdisciplinaire en santé et en sciences humaines et sociales : apports d'un séminaire de recherche », Santé publique, $\mathrm{n}^{\circ}$ 5, vol. 25, p. 579-586.

Klawiter, M. 2004. "Breast cancer in two regimes: The impact of social movements on illness experiences », Sociology of Health and Illness, vol. 26, n 6, p. 845-874.

Knobé, S. 2015. «Une publicisation entre-soi: quand les patients atteints de cancer prennent la parole », dans A. Meidani, É. Legrand, B. Jacques (sous la direction de), La santé : du public à l'intime, Paris, École des hautes études en santé publique, p. 69-81.

Kunkel, E. ; Bakker, J. ; Myers, R. ; Oyesablun, O. ; Gomella, L. 2000. «Biopsychosocial aspects of prostate cancer $»$, Psychosomatics, ${ }^{\circ} 41$, p. 85-94.

Lagrée, J. 2002. Le médecin, le malade et le philosophe, Paris, Bayard.

Laplantine, F. 2005. La description ethnographique, Paris, Armand Colin. Lauretis, T. de. 2007. Théorie queer et cultures populaires, Paris,

La Découverte.

Le Blanc, G. 2004, Les maladies de l'homme normal, Paris, Éditions du passant.

Le Breton, D. 2015. Disparaître de soi, Paris, Éditions Métailié.

Le Feuvre, N. 2003. Penser la dynamique du genre, thèse pour l'habilita-tion à diriger des recherches, université Toulouse 2, 2 tomes.

Le Goff, J. ; Jean-Charles, S. 1985. Les maladies ont une histoire, Paris, Le Seuil.

Legrand, É.; Meidani, A. 2018. «Les femmes salariées face aux risques toxiques pour la reproduction: genre, santé reproductive et risques professionnels », dans A. Meidani et A. Alessandrin (sous la direction de), Parcours de santé. Parcours de genre, Toulouse, Presses univer-sitaires du Midi.

Legros, M. 2009. «Étude exploratoire sur les blogs personnels santé et maladie », Santé publique, $\mathrm{n}^{\circ} 21$, p. 41-51.

Lerner, B. 2001. The Breast Cancer Wars: Hope, Fear, and the Pursuit of a Cure in Twentieth-Century America, Oxford University Press. 
Levy, J. et coll. 2011. Minorités sexuelles, Internet et santé, Québec, Presses de l'Université du Québec.

Lippel, K. 2015. Reconnaissance des cancers d'origine 379 professionnelle au Québec [en ligne: $\quad$ https://ruor.uottawa.ca/bitstream/10393/38655/1/ Reconnaissance $\% 20$ des $\% 20$ cancers $\% 20 d \% 27$ origine $\% 20$ professionnelle \%20au \%20Qu \%C3 \%A9bec.pdf].

Littlewood, R. 2001. «Nosologie et classifications psychiatriques selon les cultures: les « syndromes liés à la culture », L’autre, vol. 3, n² 2, p. 441-466.

Lochlann Jain, S. 2007a. «Cancer butch », Cultural Anthropology, vol.22, n 4, p. 501-538.

Lochlann Jain, S. 2007b. «Living in prognosis: Toward an elegiac poli-tics », Representations, vol. $98, \mathrm{n}^{\circ} 1, \mathrm{p} .77-92$.

Lochlann Jain, S. 2013. , Malignant. How Cancer Becomes Us, Berkeley, University of California Press.

Lock, M.; Young, A. ; Alberto, C. (sous la direction de). 2000. Living and Working with the New Medical Technologies: Intersections of Inquiry, Cambridge University Press.

Lorber, J.; Moore, L. 2002. Gender and the Social Construction of Illness, New York, Altamira Press.

Lorde, A. 1980, The Cancer Journals, New York, Aunt Lute Books.

Loseke D.-R. 1997. "The whole spirit of modern philanthropy: The construction of the idea of charity, 1912-1992 », Social Problems, vol. 44, n 4, p. 425-444.

Löwy, I. 2010. Preventive Strikes. Women, Precancer and Prophylactic Surgery, Baltimore, The John Hoplkins University Press.

Löwy, I. 2011. A Woman's Disease: The History of Cervical Cancer, Oxford, Oxford University Press.

Löwy, L. 2013. «Le genre du cancer », Clio. Femmes, Genre, Histoire, n 37, p. 65-83.

Lupton, D. 2012. Medecine as Culture: Illness, Disease and the Body, Londres, Sage Publications.

Malavolti, L.; Paraponaris, A.; Ventelou, B. 2008. «La reprise du travail après un diagnostic de cancer: un processus distinct entre hommes et femmes », dans A.-G., Le Corroller-Soriano, L. Malavolti,- C. Mermilliod (sous la direction de), La vie deux ans après un diagnostic de cancer, Paris, La Documentation française, Base docu-mentaire bdsp, Études et statistiques, p. 259-270.

Malet, J. 2009. «Les associations, source de vitalité du milieu rural ? », Pour, vol. 2, n 201, p. 97-102.

Mane, P. ; Aggleton, P. 2001. «Gender and hiv/aids: What do men have to do with it?» Current Sociology, vol. 6, $\mathrm{n}^{\circ}$ 49, p. 23-37.

Margolies, L. ; Brown, C. 2018, «Current state of knowledge about cancer in lesbians, gay, bisexual and transgender (lgbt) people », Seminars in Oncology Nursing, vol. 34, $n^{\circ} 1$, p. $3-$ 11.

Marino, P. ; Sagaon Teyssier, L. ; Laetitia, M. ; Le Corroler--Soriano, A.-G. 2013. «Sex differences in the return-to-work process of cancer survivors 2 years after diagnosis: Results from a large french popu-lation-based sample », Journal of Clinical Oncology, vol. $31, \mathrm{n}^{\circ} 10$, p. 1277- 1284 [en ligne : https://doi.org/10.1200/JCO.2011.38.5401. 388]. 
Marshall, B.; Katz, S. 2002. «Forever functional: Sexual fitness and the ageing male body », Body and Society, vol. 8, n 43, p. 43-70.

Martro, E. et coll. 2007. "Risk factors for human herpesvirus 8 infec-tion and aidsassociated Kaposi's sarcoma among men who have sex with men in a European multicentre study », International Journal of Cancer, $n^{\circ} 120$, p. 1129-1135.

Maruani, M. 2013. Travail et genre dans le monde, Paris, La Décou-verte [en ligne : https://www.cairn.info/travail-et-genre-dans-le-monde-9782707174567.htm. 390].

Maruani, M. 2017. Travail et emploi des femmes, Paris, La Découverte. Massé, R. 1995. Culture et santé publique. Les contributions de l'anthropo-

logie à la prévention et la promotion de la santé, Montréal, Éditions Gaëtan Morin.

Massé, R. 2007. «Les sciences sociales face au défi de la santé publique », Sciences sociales et santé, vol. $25, \mathrm{n}^{\circ} 1$, p. 5-23.

Massé, R. 2008. Détresse créole. Ethno-épidémiologie de la détresse psychique à la Martinique. Québec, Pul.

Mathieu, N.-C. 1985. «Quand céder n'est pas consentir. Des détermi-nants matériels et psychiques de la conscience dominée des femmes et de quelques-unes de leurs interprétations en ethnologie », dans N.-C. Mathieu (sous la direction de), L'arraisonnement des femmes. Essais en anthropologie des sexes, Paris, éditions de l'École des hautes études en sciences sociales, p. 169-245.

Mathieu, N.-C. 1991. L'anatomie politique. Catégorisations et idéologies du sexe, Paris, Indigo \& Côtés-femmes.

Mauss, M. 2009. «Les techniques du corps », dans M. Mauss, Sociologie et anthropologie, Paris, Puf, p. 363-386.

Mccall, L. 2005. «The complexity of intersectionality », Signs, vol. 30, n 3, p. 1771-1800.

Meeuwesen, L. ; Bensing, J.; Van Den Brink-Muinen, A. 2002. «Communicating fatigue in general practice and the role of gender », Patient Education and Counseling, $\mathrm{n}^{\circ} 48$, p. 233-242.

Meidani, A. (à paraître). «Cancer, soutien social et genre: des réseaux personnels mobilisés dans le cas de la maladie grave », Sciences sociales et santé (accepté).

Meidani, A. 2007. Cancers et genre: des enjeux de prévention à l'adhésion aux soins, Rapport I pour l'inca.

Meidani, A. 2008. «Le genre dans l'histoire de la maladie cancéreuse : le façonnement sociétal de la santé et ses inégalités », Sociologie santé, n²8, p. 81-120.

Meidani, A. 2018. «Le cancer a-t-il un genre? », dans A. Meidani et A. Alessandrin (sous la direction de), Parcours de santé. Parcours de genre, Toulouse, Presses universitaires du Midi.

Meidani, A. ; Alessandrin, A. 2017. «Cancers et transidentités : une nouvelle population à risques? », Sciences sociales et santé, vol. $35, n^{\circ} 1$, p. 41-64.

Meidani, A. ; Alessandrin, A. (sous la direction de). 2018. Parcours de santé, parcours de genre, Toulouse, Presses universitaires du Midi.

Meidani, A. ; Alessandrin, A. 2019. «Quand le cancer rencontre le genre », Revue française de sociologie, vol. $60, \mathrm{n}^{\circ} 2$, p. 201-224. 
Meidani, A. ; Alessandrin, A. 2019. «La fabrique des corps sexués. Entre médicalisation et normalisation. La place du corps dans les trans studies en France », dans H. Martin et M. Roca i Escoda (sous la direction de), Sexuer le corps. Huit études sur les pratiques médicales d'hier et d'aujourd'hui, Lausanne, Éditions hetsl [en ligne].

Meidani, A. ; Cavalli S (sous la direction de). 2019. Figures du vieillir et formes de déprise, Toulouse, érès.

Meidani, A. ; Legrand, É. ; Jacques, B. (sous la direction de). 2015. La santé : du public à l'intime, Paris, École des hautes études en santé publique.

Membrado, M. 2006. «Les femmes dans le champ de la santé : de l'oubli à la particularisation $»$, Nouvelles questions féministes, vol. 25, n² 2 , p. 16-31.

Ménoret, M. 1999. Les temps du cancer, Paris, Éditions du cnrs.

Menvielle, G.; Chastang, J.-F.; Luce, D.; Leclerc, A. ; pour le groupe edisc. 2007. «Évolution temporelle des inégalités sociales de mortalité en France entre 1968 et 1996. Étude en fonction du niveau d'études par causes de décès », Revue d'épidémiologie et santé publique, vol. $55, \mathrm{n}^{\circ} 2$, p. 97-105.

Meslé, F. 2004. «Écart d'espérance de vie entre les sexes: les raisons du recul de l'avantage féminin », Revue d'épidémiologie et de santé publique, vol. 52, n 4, p. 333-352.

Meyer, D. 2012. «An intersectional analysis of lesbian, gay, bisexual, and transgender (lgbt) people's evaluations of anti-queer violence », Gender and Society, vol. 26, n 6 , p. 849-873.

Miller, E. 1986. The Marginalization of the Black Male: Insights from the Development of the Teaching Profession, Kingston, Jamaica, Institute of Social and Economic Research.

Miller, E. 1991. Men at Risk, Kingston, Jamaica Publishing House.

Mol, A. 2002. The Body Multiple: Ontology in Medical Practice, Durham et Londres, Duke University Press.

Molinier, P. 2012. "Chirurgie : une mise à distance nécessaire des émotions? », Sciences sociales et santé, $n^{\circ} 30$, p. 99-103.

Molinier, P. 2016. «De la civilisation du travail à la société du care », Vie sociale, vol. 2, $\mathrm{n}^{\circ} 14$, p. $127-140$.

Molinier, P. ; Laugier, S. ; Paperman, P. 2009. Qu'est-ce que le care ?, Paris, Payot.

Mongiat-Artus, P. ; Peyromaure, M. ; Richaud, P. ; Droz, J.-P. ; R-ainfray, M. ; Jeandel, C. ; Rebillard, X. ; Moreau, J.-L. ; Davin, J.-L. ; Salomon, L. ; Soulié, M. ; les membres du souscomité prostate du comité cancérologie-association fran-çaise d'urologie. 2009. « Recommandations pour la prise en charge du cancer de la prostate chez l'homme âgé : un travail du comité de cancérologie de l'association française d'urologie», Progrès en urologie, vol. $19, \mathrm{n}^{\circ} 11, \mathrm{p} .810-817$.

Morin, E. 1973. Le paradigme perdu: la nature humaine, Paris, Le Seuil. Morin, T.; Remila, N. 2013. «Le revenu salarial des femmes reste infé-rieur à celui des hommes », insee, $\mathrm{n}^{\circ} 1436$, [en ligne : https://www.

insee.fr/fr/statistiques/1280986].

Moynihan, C. 2002. «Men, women, gender and cancer», European Journal of Cancer Care, vol. $11, \mathrm{n}^{\circ} 3$, p. 166-172.

Nancy, J.-L. 2000. L’intrus, Paris, Éditions Galilée. 
Nengeh Mensah, M. 2016. «Le témoignage comme stratégie d'inter-vention sociale et culturelle : l'expérience de personnes vivant avec le vih/sida », Revue Intervention, $\mathrm{n}^{\circ} 143$, p. 33-48.

Newton, E. 2008. «Le mythe de la lesbienne masculine: Radclyffe Hall et la Nouvelle Femme », Cahiers du Genre, n 45, p. 15-42.

Nichter, M.; Lock, M. (sous la direction de). 2002. New Horizons in Medical Anthropology, Londres, Routledge.

Nolte, K. 2008. "'Carcinoma uteri' and 'debouchery-morality, cancer and gender in the nineteenth century' », Social History of Medicine, vol. 21, n 1, p. 31-46.

Novas, C. ; Nikolas, R. 2000. "Genetic risk and the birth of the somatic individual», Economy and Society, vol. 29, $n^{\circ} 4$, p. 485-513.

O’Brien, R. ; Hunt, K. ; Hart, G. 2007. «Standing out from the Herd: Men Renegotiating Masculinity in Relation to their Experience of Illness », International Journal of Men's Health, vol. 6, n 3, p. 178-200.

O’grady, H. 2005. Woman's Relationship with Herself: Gender, Foucault and Therapy, New York, Routledge.

Obedin-Maliver, J. ; Goldsmith, E. ; Stewart, L. ; White, W. ; Tran, E. ; Brenman, S. ; Wells, M. ; Fetterman, D. ; Garcia, G. ; Lunn, M. 2011. «Lesbian, gay, bisexual and transgenderrelated content in undergraduate medical education », Journal of the American Medical Association, vol. 306, n 9, p. 971-977.

Observatoire sociétal des cancers. 2014. Cancer du sein. Se recons-truire après une mastectomie, La ligue, Rapport 2014, [en ligne: https://www.liguecancer.net/sites/default/files/docs/observatoire_societal_des_cancers_rapport_2014.pdf]

Oliffe, J.-L. 2011. «Positioning prostate cancer as the problematic third testicle », dans A. Broom et P. Tovey (sous la direction de), Men's Health and Illness. Body, Identity and Social Context, West Sussex, Wiley-Blackwell, p. 33-62.

Ortner, S.-B. 1972. «Is female to male as nature is to culture?», Femi-nist Studies, vol. 1, $\mathrm{n}^{\circ} 2$, p. $5-31$.

Pace, E. 1993. «Le retour du sacré », dans J.-A. Beckford et D. Hervieu-Léger (sous la direction de), Religion et écologie, Paris, Le Cerf, p. 197-213.

Paiva, M. 2012. «Des femmes invisibles », Plein droit, vol. 2, n 93, p. 21- 24. [en ligne : https://doi.org/10.3917/pld.093.0021].

Paiva, M. 2016. «De l'invisibilité des cancers d'origine profession-nelle à l'invisibilisation des risques cancérogènes dans le travail des femmes. Une analyse à partir de l'enquête permanente du Grou-pement d'intérêt scientifique sur les cancers d'origine professionnelle-giscop93 », thèse de doctorat en sociologie, Paris, université Paris 8.

Parsons, T. 1991. The Social System, Londres, Routledge.

Pattison, S., McLaren, B. 2013. "Triple negative breast cancer in a male to female transsexual », Internal Medicine Journal, vol. 43, n 2, p. 203-205.

Patton, C. 2007. "Mobile knowledge: hiv patients' encounters with endocrinology ", Canadian Journal of Communication, vol. 32, $\mathrm{n}^{\circ} 3 / 4$, p. 335-355.

Paul, L. et coll., 2014. «Support needs and resources of sexual mino-rity women with breast cancer », Psycho-Oncology, vol. 23, n 5 , p. 578-584. 
Peitzmeier, S.-M. ; Reisner, S.-L. ; Harigopal, P. ; Potter, J. 2014. «Female-to-male patients have high prevalence of unsatisfac-tory paps compared to non-transgender females: Implications for cervical cancer screening », Journal of General Internal Medicine, vol. 29, $\mathrm{n}^{\circ} 5$, p. $778-784$.

Peretti-Wattel, P. 2001. La société du risque, Paris, La Découverte.

Peterson, S. 2003. A Critical Rewriting of Global Political Economy: Inte-grating Reproductive, Productive and Virtual Economies, Londres, Routledge.

Piccand, L. 2013. «Du syndrome prémenstruel au trouble dyspho-rique prémenstruel. Façonnements locaux de catégories diagnos-tiques et reconfiguration des représentations sur la vulnérabilité des femmes », dans F. Knittel et P. Raggi (sous la direction de), Genre et techniques. xixe-xxie siècles, Presses universitaires de Rennes, p. 141-151.

Pierret, J. 2006. Vivre avec le vih. Enquête de longue durée auprès des personnes infectées, Paris, Puf.

Pierret, J. 2007. «Une analyse en termes de carrières : le cas de personnes traitées par des antirétroviraux hautement actifs contre le vih entre 1998 et $2000 »$, Sciences sociales et santé, vol. 25, n² 2, p. 73-99.

Pillot, A. 1992. Vivre le cancer. Étude psychosociologique de l'univers des malades atteints de cancer, thèse de sociologie, Grenoble, université Pierre-Mendès-France.

Plummer, D.; McLean, A. ; Simpson, J. 2008. «Has learning become taboo and is risktaking compulsory for caribbean boys ? Researching the relationship between masculinities, education and risk ", Caribbean Review of Gender Studies, $\mathrm{n}^{\circ} 2$ [en ligne: https:// sta.uwi.edu/crgs/september2008/abstracts/DPlummerAMcleanJ-Simpson.pdf].

Polite, B.-N.; Adams-Campbell, L.; Brawley, O.-W. ; Bickell, N. ; Carethers, J.-M. ; Flowers, C.-R.; Foti, M. et coll. 2017. «Char-ting the future of cancer health disparities research: A position statement from the American Association for Cancer Research, the American Cancer Society, the American Society of Clinical Onco-logy, and the National Cancer Institute », Journal of Clinical Onco-logy, vol. 35, n² 26, p. 3075-3082.

Pollak, M. 1988. Les homosexuels et le sida. Sociologie d'une épidémie, Paris, Métailié.

Potts, A.; Tiefer, L. (sous la direction de). 2006. Sexualities, vol. 9, n 3, London, Sage Publications.

Prearo, M. 2014. Le moment politique de l'homosexualité. Mouvements, identités et communautés en France, Presses universitaires de Lyon.

Preos [Prévenir le rejet basé sur l'orientation sexuelle et l'identité de genre chez les jeunes]. 2012. Rapport du groupe santé, Vers l'égalité des chances en matière de santé pour les personnes lgbt : le rôle du système de santé. État des lieux et recommandations [en ligne].

Printz, C. 2015. «Risk assessment: Physicians discuss motivations for rising rates of prophylactic mastectomies », Cancer, vol. 121, n 11, p. 1721-1722.

Pujol, P. 2012. «État de l'art des recommandations actuelles pour la chirurgie prophylactique », dans M. Namer, M. Héry, M. Spielmann, J. Gligorov, F. Penault-Llorca (sous la direction de), Cancer du sein. Compte rendu du 12e cours supérieur francophone de cancérologie, Nice/Saint-Paul-de-Vence, Springer.

Quinn, G. 2015a. «Cancer and lesbian, gay, bisexual, transgender/Trans-sexual, and queer/questioning populations (lgbtq)», Canadian Cancer Journal Clinic, vol. 65, $\mathrm{n}^{\circ} 5$, p. 384-400. 
Quinn, G. 2015b. «The importance of disclosure: lesbian, gay, bisexual, transgender/transsexual, queer/questioning, and intersex individuals and the cancer continuum », Cancer, vol. 121, n 8 , p. 1160-1163.

Rabinow, P. 1996. Artificiality and Enlightenment: From Socio-Biology to Biosociality. Essays on the Anthropology of Reason, Princeton Univer-sity Press, p. 91-111.

Radcliffe, S. ; Laurie, N. ; Andolina, R. 2004. " The transnationaliza-tion of gender and reimagining Andean indigenous development », Signs, ${ }^{\circ}$ 29, p. 387-416.

Rail, G. ; Bryson, M.-K. ; Hart, T.-L. ; Gahagan, J. ; Ristock, J. 2017. «Proyecto Cancer's Margins: minorías sexuales, asistencia oncológica, conocimiento y subjetividades », dans A. Porroche-- Escudero; G. Coll-Planas et C. Riba (sous la direction de), Cica-trices (in)visibles: Perspectivas feministas sobre el cáncer de mama, Barcelona, Edicions Bellaterra, p. 71-82.

Rail, G. ; Bryson, M.-K. ; Hart, T.-L. ; Gahagan, J. ; Ristock, J. 2019. « Artivisme lesbien et queer en marge du cancer: de l'importance des savoirs subjugués », dans D. Bourque et J. Coulombe (sous la direc-tion de), artivismes lesbiens à l'ère de la mondialisation, Montréal, Les Éditions sans fin [Never Ending Press].

Rail, G. ; Molino, L. ; Fusco, C. ; Norman, M.-E. ; Petherick, L. ; Polzer, J. ; Moola, F. ; Bryson, M. 2018. " hpv vaccination campaings and the discursive construction of "at-risk" girls », Cana-dian Journal of Public Health, vol. 109, n 5, p. 622-632.

Richard, G. 2015. «The pedagogical practices of Québec high school teachers relative to sexual diversity », Journal of lgbt Youth, vol. 12, n² 2, p. 113-143.

Riese, W. 1953. The Conception of Disease: Its History, Its Versions, and Its Nature, New York, Philosophical Library.

Robertson, A. 2001. «Biotechnology: Political rationality and discourses on health risk », Health, vol. 5, n 3, p. 293-309.

Rosario, M.; Schrimshaw, E. ; Hunter, J. 2008. «Butch/femme diffe-rences in substance use and abuse among young lesbian and bisexual women: Examination and possible explanations », Substance Use and Misuse, $n^{\circ} 43$, p. 1002-1015.

Rosende, M.; Schoeni, C. 2012. «Seconde partie de carrière, régime de retraite et 399 inégalités de sexe », Revue française des affaires sociales, ${ }^{\circ} 2$, p. 130- 147.

Rosenfeld, D.; Faircloth, C. 2006. Medicalized Masculinities, Philadel-phia, Temple University Press.

Rouëssé, J. 2011. Une histoire du cancer du sein en Occident : enseigne-ments et réflexions, Paris, Springer.

Rozet, F.; Hennequin, C.; Beauval, J.-B.; Beuzeboc, P.; Cormier, L. ; Fromont, G. ; Mongiat-Artus, P.; Ouzzane, A.; Ploussard, G. ; Azria, D. ; Brenot-Rossi, I. ; CancerlTassin, G. ; Cussenot, O.; Lebret, T.; Rebillard, X. ; Soulié, M. ; Renard-Penna, R. ; Méjean, A. 2016. "Recommandations en onco-urologies 2016-2018 du Comité de cancérologie de l'Association française d'urologie, cancer de la prostate », Progrès en urologie, vol. $27, \mathrm{n}^{\circ} 1$, p. 95-143.

Ruault, L. 2016. «La circulation transnationale du self-help féministe : acte 2 des luttes pour l'avortement libre? », Critique internationale, vol. 70, $\mathrm{n}^{\circ} 1$, p. 37-54.

Salle, M. ; Vidal, C. 2017. Femmes et santé : encore une affaire d'homme ?, Paris, Belin. 
Scanlon, M.; Blaes, A.; Geller, M. ; Majhail, N.-S.; Lindgren, B. ; Haddad, T. 2012. «Patient satisfaction with physician discussions of treatment impact on fertility, menopause and sexual health among pre-menopausal women with cancer », Journal of Cancer, vol. 3, $\mathrm{n}^{\circ} 1$, p. $217-225$.

Scheim, A.-I. ; Bauer, G.-R. 2013. Breast and Cervical Cancer Screening Among Trans Ontarians, Toronto, Trans pulse and the Canadian Cancer Society.

Scheper-Hughes, N. ; Lock, M.-M. 1987. «The mindful body: A prole-gomenon to future work in medical anthropology », Medical Anthro-pology Quarterly, vol. 1, n 1, p. 6-41.

Schiebinger, L. 1993. Nature's Body. Gender in the Making of Modern Science, New Brunswick, NJ, Rutgers University Press.

Schmittdiel, J.; Grumbach, K. ; Selby, J. ; Quesenberry, C. 2000. «Effect of physician and patient gender concordance on patient satisfaction and preventive care practice », Journal of General Internal Medicine, $\mathrm{n}^{\circ}$ 15, p. 761-769.

Sentis, I. 2020. "Mon corps, moi-même », dans A. Alessandrin, J. Dagorn, A. Meidani, G. Richard et M. Toulze (sous la direction de), Santé LGBT, Bord de l'eau.

Serviant-Fine, T. 2017, Une approche rationnelle de la chimiothérapie: histoire des antimétabolites (1935-1955), thèse d'épistémologie et histoire des sciences et des techniques, Lyon, université Claude-- Bernard Lyon 1.

Sinding, C. ; Barnoff, L. ; Grassau, P. 2004. «Homophobia and hetero- sexism in cancer care: The experiences of lesbians "), Canadian Journal of Nursing Research, vol. 36, $\mathrm{n}^{\circ} 4$, p. 171-188.

Sinding, C. ; Grassau, P. ; Barnoff, L. 2006. « Community support, community values: The experiences of lesbians diagnosed with cancer $»$, Women and Health, vol. 44, $n^{\circ} 2$, p. 59 79.

Skloot, R. 2010. The Immortal Life of Henrietta Lacks, New York, Crown Publishers.

Spurlin, W. 2018. «Queer theory and biomedical practice: The bio- medicalization of sexuality/the cultural politics of biomedicine », Journal of Medical Humanities [en ligne : https://www.ncbi.nlm.nih. gov/pubmed/30073625].

Stratonovitch, C. 2009. "Genre et médecine », Chimères, vol. 1, n 69, p. 135-147 [en ligne : https://www.cairn.info/revue-chimeres-2009-1-page-135.htm].

Strauss, A. 1992. La trame de la négociation, textes réunis et présentés par I. Baszanger, Paris, L'Harmattan.

Strauss, A.-L; Corbin, J.; Fagerhaugh, S.; Glaser, B.-G. ; Maines, D. ; Suczeck, B. ; Wiener, C.-L. 1975. Chronic Illness and the Quality of Life, Saint-Louis, C.V. Mosby Co.

Street, R. 2002. «Gender differences in health care provider-patient communication: Are they due to style, stereotypes, or accommoda-tion? », Patient Education and Counseling, $\mathrm{n}^{\circ} 48$, p. 201-206.

Sulik, G. 2011. Pink Ribbon Blues. How Breast Cancer Culture Under-mines Women's Health, Oxford University Press.

Tarantini, C.; Gallardo, L.; Peretti-Watel, P. 2014. «Travailler après un cancer du sein. Enjeux, contraintes et perspectives », Socio-logie, vol. 5, n² 2, p. 139-155.

Tavris, C. 1992. The Mismeasure of Woman: Why Women Are Not the Better Sex, the Inferior Sex, or the Opposite Sex, Londres, Reprint Edition, Kindle Edition 
Taylor, E.-T. ; Bryson, M.-K. 2016. «Cancer's margins: Trans and gender nonconforming people's access to knowledge, experiences of cancer health, and decision-making », lgbt Health, vol. $3, \mathrm{n}^{\circ} 1$, p. $79-89$.

Temkin, O. 1945. The Falling Sickness: A History of Epilepsy from the Greeks to the Biginnings of Modern Neurology, Baltimore, John Hopkins Press.

Théry, I. 2016. Mariage et filiation pour tous. Une métamorphose inachevée, Paris, Le Seuil.

Thiaudière, C. 2002. Sociologie du sida, Paris, La découverte.

Thiers-Vidal, L. 2002. «De la masculinité à l'anti-masculinisme: penser les rapports sociaux de sexe à partir d'une position sociale oppres-sive», Nouvelles questions féministes, vol. $21, \mathrm{n}^{\circ} 3$, p. 71-83.

Thompson, C. 2005. Making Parents: The Ontological Choreography of

Reproductive Technologies, Cambridge, Massachusetts Institute of

Technology Press.

Tiefer, L. 1994. «The medicalization of sexuality », Annual Review of Sex Research, $n^{\circ} 7$, p. 252-282.

Tjepkema, M. 2008. "Health care use among gay, lesbian and bisexual Canadians. Statistics Canada, Catalogue 82-003 », Health Reports, vol. 19, n 1, p. 53-64.

Toulze, M.; Meidani, A. (sous la direction de). 2017. "Santé et discriminations », Les cahiers de la lcd-Lutte contre les discriminations, vol. 3, $\mathrm{n}^{\circ} 5$, p. 13-24.

Tresvaux du Fraval, F. 2013. Le dispositif d'annonce du cancer : reflet de l'évolution de la place du patient dans le système sanitaire français, thèse d'épistémologie et histoire des sciences et des techniques, Paris, université Paris 7.

Trigoni, M.; Griffiths, F.; Tsiftsis, D. et coll. 2008. «Mammography screening: Views from women and primary care physicians in Crete », bmc Women's Health, vol. $8, \mathrm{n}^{\circ} 20$, p. 8-20, [en ligne : https:// doi.org/10.1186/1472-6874-8-20].

Tronto, J. 1993. Moral Boundaries. A Political Argument For an Ethic of Care, New YorkLondres, Routledge.

Tuppin, P. ; Leboucher, C. ; Dougé, M. ; Peyre-Lanquar, G. ; Gabach, P. ; Descotes, J.-L. ; Rebillard, X. 2016. «Dépistage indi-viduel du cancer de la prostate chez les hommes de 40 ans et plus, France, 2009-2015. Données du système national d'information interrégimes de l'assurance maladie », Bulletin épidémiologique hebdomadaire, $\mathrm{n}^{\circ} 39-40$, p. 700-706.

Ussher, J. 2013. «The experience and construction of changes to women's sexuality after breast cancer », dans D. Castaneda (sous la direction de), The Essential Handbook of Women's Sexuality, Londres, Praeger, p. 171-195.

Ussher, J. 2017. «Let's talk about gay sex: Gay and bisexual men's sexual communication with healthcare professionals after prostate cancer », European Journal of Cancer Care, vol. $26, \mathrm{n}^{\circ} 1, \mathrm{p} .1-12$.

Vega, A. 2012. » La mort, l'oubli et les plaisirs. Les cheminements des patientes dans le cancer du sein », Anthropologie et santé, n 4 [en ligne : https://journals.openedition.org/anthropologiesante/861].

Vidal, L. 1999. «Anthropologie d'une distance. Le sida, de réalités multiples en discours multiformes », Autrepart, n 12, p. 19-36. 
Vidal, L. 2000. «De connaissances en pratiques. Évaluer les risques du sida », dans J.-P. Dozon et D. Fassin (sous la direction de), Critique de la santé publique, Paris, Balland, p. 233-256.

Voegtli, M. 2004. «Du jeu dans le Je: ruptures biographiques et travail de mise en cohérence », Lien social et politique, $n^{\circ} 51$, p. 145-158.

Waldby, C. 1996. Aids and the Body Politic: Biomedicine and Sexual Difference, Londres, sage Publications.

Wall, D. ; Kristjanson, L. 2005. «Men, culture and hegemonic mascu-linity: Understanding the experience of prostate cancer $»$, Nursing Inquiry, vol. $12, n^{\circ} 2$, p. $87-97$.

Wassersug, R.-J.; Oliffe, J.-L. 2009. «The social context for psycho-logical distress from iatrogenic gynecomastia with suggestions for its management», The Journal of Sexual Medicine, vol. 6, $\mathrm{n}^{\circ} 4$, p. 989-1000.

Weil-Olivier, C. 2008. Vie de femmes : 50 ans d'avancées médicales, Montrouge, J. Libbey Eurotext.

Yalom, M. 1997. A History of the Breast, New York, Ballantines Book. Young, I.-M. 1992. «Breasted experience: The look and the feeling », dans D. Leder, On Female Experience: 'Throwing Like a Girl' and Other Essays, Oxford University Press, p. 215-230.

Zahm, S.-H. ; Blair, A. 2003. «Occupational cancer among women: Where have we 401 been and where are we going? », American Journal of Industrial Medicine, vol. 44, $\mathrm{n}^{\circ} 6$, p. 565- 575 [en ligne : https://doi.org/10.1002/ajim.10270].

Ziebland, S.; Herxheimer, A. 2008. «How patients' experiences contribute to decision making: Illustrations from dipex », Journal of Nursing Management, vol. 16, n 4, p. 433 439.

Zolesio, E. 2012. Chirurgiens au féminin? Des femmes dans un métier d'hommes, Presses universitaires de Rennes. 